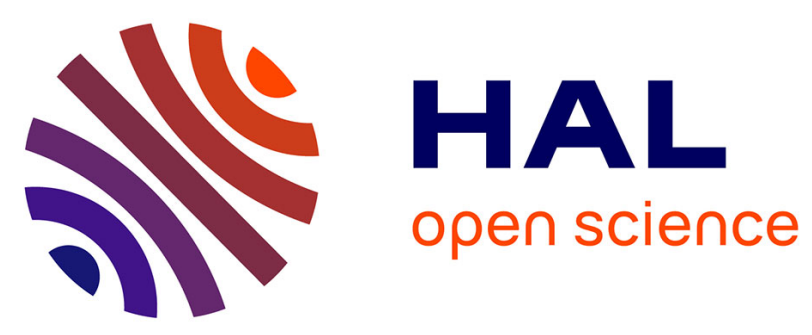

\title{
Late Paleozoic strike-slip shear zones in eastern central Asia (NW China): New structural and geochronological data
}

Sébastien Laurent-Charvet, Jacques Charvet, Patrick Monié, Liangshu S. Shu

\section{- To cite this version:}

Sébastien Laurent-Charvet, Jacques Charvet, Patrick Monié, Liangshu S. Shu. Late Paleozoic strikeslip shear zones in eastern central Asia (NW China): New structural and geochronological data. Tectonics, 2003, 22, pp.2, 1009. 10.1029/2001TC901047 . hal-00091070

\section{HAL Id: hal-00091070 \\ https://hal-insu.archives-ouvertes.fr/hal-00091070}

Submitted on 28 Jul 2015

HAL is a multi-disciplinary open access archive for the deposit and dissemination of scientific research documents, whether they are published or not. The documents may come from teaching and research institutions in France or abroad, or from public or private research centers.
L'archive ouverte pluridisciplinaire HAL, est destinée au dépôt et à la diffusion de documents scientifiques de niveau recherche, publiés ou non, émanant des établissements d'enseignement et de recherche français ou étrangers, des laboratoires publics ou privés. 


\title{
Late Paleozoic strike-slip shear zones in eastern central Asia (NW China): New structural and geochronological data
}

\author{
Sébastien Laurent-Charvet and Jacques Charvet \\ Institut des Sciences de la Terre d'Orléans, Université d'Orléans, Orléans, France
}

Patrick Monié

Unité Mixte de Recherche 5573, Université des Sciences et Techniques du Languedoc, Montpellier, France

Liangshu Shu

Department of Earth Sciences, University of Nanjing, Nanjing, China

Received 19 November 2001; revised 10 April 2002; accepted 16 July 2002; published 2 April 2003.

[1] New structural studies and ${ }^{40} \mathrm{Ar} /{ }^{39} \mathrm{Ar}$ dating in northwest China provide information about late Paleozoic strike-slip motions subsequent to accretional events, which built eastern central Asia during the Paleozoic. Two principal areas were affected by these large transcurrent motions. First, in the Tianshan range, main east-west ductile shear zones are dextral and coeval with an eastward decreasing greenschist retrograde metamorphism. Associated biotites give ages ranging from $290 \mathrm{Ma}$ to $245 \mathrm{Ma}$. The earlier N110 shearing occurred in western Tianshan, while the last one, dextral in whole Tianshan, occurred 250-245 Myr ago. Second, in the Chinese Altay region several NW-SE shear zones structured the area. The main motion is sinistral and occurred along the Erqishi zone at 280-290 Ma. It is followed by a complex succession of dextral and sinistral shearing episodes, leading to the northwestward structuring, dated at 245 $\mathrm{Ma}$, of a metamorphic zone that was folded during a compressive event. INDEX TERMS: 8110 Tectonophysics: Continental tectonics - general (0905); 9320 Information Related to Geographic Region: Asia; 1035 Geochemistry: Geochronology; KEYWORDS: central Asia, Paleozoic, strike-slip, structures, geochronology. Citation: Laurent-Charvet, S., J. Charvet, P. Monié, and L. Shu, Late Paleozoic strike-slip shear zones in eastern central Asia (NW China): New structural and geochronological data, Tectonics, 22(2), 1009, doi:10.1029/ 2001TC901047, 2003.

\section{Introduction}

[2] Following successive accretions to the southern margin of Eurasia of continental blocks, arcs, and accretionary complexes that form the huge orogenic collage named the

Copyright 2003 by the American Geophysical Union. 0278-7407/03/2001TC901047
Altaids [Natal'in and Sengör, 1994; Sengör et al., 1993], central Asia suffered late Paleozoic large-scale transcurrent events responsible for the dominant structures in this area [Allen et al., 1993; Laurent-Charvet et al., 2002; Sengör et al., 1993; Sengör and Natal'in, 1996]. In NW China the Xinjiang province, which is bordered by Kazakhstan, Siberia, and Mongolia (Figure 1), is a key region for understanding the late Paleozoic history of central Asia. The Tianshan orogenic belt, which extends over more than 3000 $\mathrm{km}$ in an east-west direction, is one of the most important mountain ranges of this part of central Asia. It separates the Junggar block to the north from the Tarim block to the south and has been formed during two main accretionary events (Figure 1).

[3] The present day geological division of the Tianshan orogen into three main units, South, Central, and North Tianshan [Coleman, 1989; Ma et al., 1990; Windley et al., 1990], results from the late Paleozoic strike-slip shear zones. Farther north, in the Chinese Altay Mountains, important strike-slip shear zones such as the Irtysh-Erqishi sinistral shear zone that runs continuously from NE Kazakhstan to north Xinjiang [Melnikov et al., 1997, 1998; Mitrokhin et al., 1997] (Figure 1) have also strongly modified the architecture of the area. During the last 20 years, Paleozoic accretion and Cenozoic deformation in Xinjiang were well documented by several studies [e.g., Allen et al., 1999; Avouac et al., 1993; Berzin et al., 1994; Burtman, 1975; Charvet et al., 2000; Coleman, 1989; Dobretsov et al., 1995; Gao et al., 1998; Hendrix et al., 1994; Ma et al., 1990; Sengör et al., 1993; Shu et al., 1999b, 2000; Windley et al., 1990]. Some authors have paid some attention to the transcurrent motions [Allen et al., 1993, 1995; Chen, 1994; Cui, 1995; Dehandschutter et al., 1997; Laurent-Charvet et al., 2000a, 2002; Liu et al., 1996; Ma et al., 1997; Melnikov et al., 1997; Mitrokhin et al., 1997; Sengör et al., 1993; Shu et al., 1999a], providing structural and geochronological information on these late Paleozoic-early Mesozoic deformations in central Asia.

[4] The purpose of this paper is to describe the structures and microstructures of ductile strike-slip faults around 


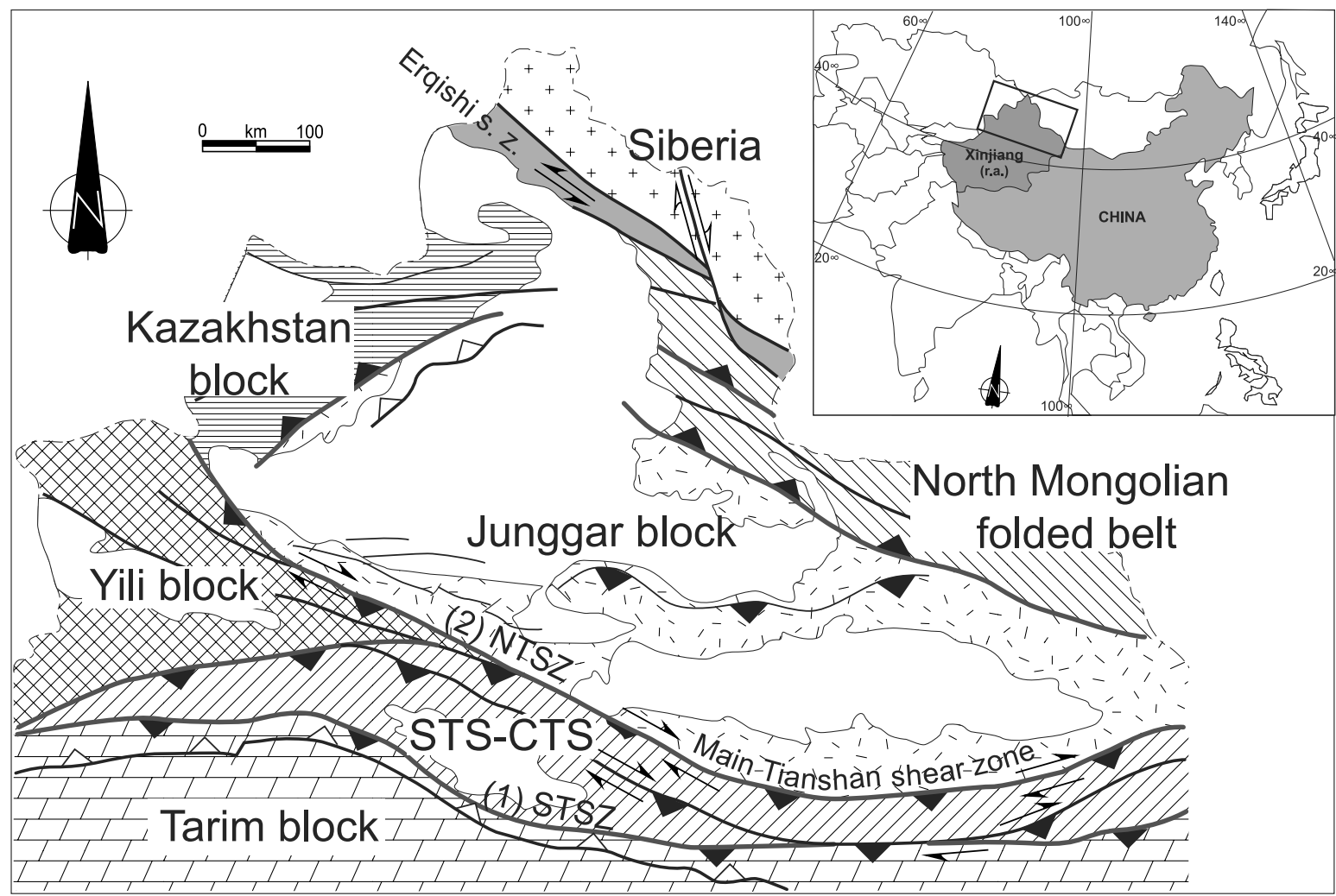

Figure 1. Simplified structural map showing the blocks that constitute the north Xinjiang (modified from Allen et al. [1992]). Abbreviations are as follows: STS-CTS, south Tianshan and central Tianshan; NTSZ, North Tianshan Suture Zone; STSZ, South Tianshan Suture Zone; and Erqishi s. z., Erqishi shear zone.

the Junggar basin and to present new ${ }^{40} \mathrm{Ar} /{ }^{39} \mathrm{Ar}$ laser probe data that provide important information on the late Paleozoic multistage activity of these faults. Two domains on the edge of the basin were studied (Figure 2): the first in the Tianshan range, between Urumqi, Hejing, and Tuokexun (between $86^{\circ} 30^{\prime} \mathrm{E}$ and $89^{\circ} \mathrm{E}$ and $42^{\circ} \mathrm{N}$ and $43^{\circ} 30^{\prime} \mathrm{N}$ ), and the second in the Chinese Altay Mountains, near Fuyun (between $89^{\circ} 15^{\prime} \mathrm{E}$ and $89^{\circ} 45^{\prime} \mathrm{E}$ and $46^{\circ} 52^{\prime} \mathrm{N}$ and $47^{\circ} 10^{\prime} \mathrm{N}$ ). Data come from our fieldwork in the whole north Xinjiang area and from Chinese and international sources. Microstructural analyses were made in Orléans (France) and Nanjing (China) Universities, and ${ }^{40} \mathrm{Ar} /{ }^{39} \mathrm{Ar}$ dating took place in Montpellier (France) and Nanjing (China) Universities. The new results are synthesized in a discussion in which we propose that relative rotations between the Tarim, Junggar, and Siberian blocks in the late Paleozoic were responsible for large-scale transcurrent motions in central Asia (e.g., the Irtysh-Erqishi shear zone), allowing the accommodation of a final shortening between these blocks.

\section{Geological Setting}

\subsection{Tianshan Range}

[5] The Xinjiang province in northwest China is located between Mongolia and Kazakhstan, in central Asia (Figure 1). The large basins of Tarim and Junggar, to the south and to the north, respectively, are separated by the east-west trending Tianshan Mountains extending from Uzbekistan and west Kazakhstan to the eastern edge of Xinjiang. In northeastern Junggar the Xinjiang province is bordered by the Altay Mountains, which stretch between Siberia and Outer Mongolia countries.

[6] The studied area includes parts of the Siberian, Junggar, Tarim, and Kazakhstan blocks [Berzin et al.,

Figure 2. (opposite) Synthetic geological map of eastern Junggar (modified from the geological map of Xinjiang Uygur Autonomous Region, scale 1:2,000,000 [Xinjiang Bureau of Geology and Mineral Resources, 1992] and 1:200,000 scale Landsat scenes). Locations of Figures 3 and 6 are shown. Sections a and b depict schematic cross sections of Tianshan (modified from Laurent-Charvet et al. [2000b]). 


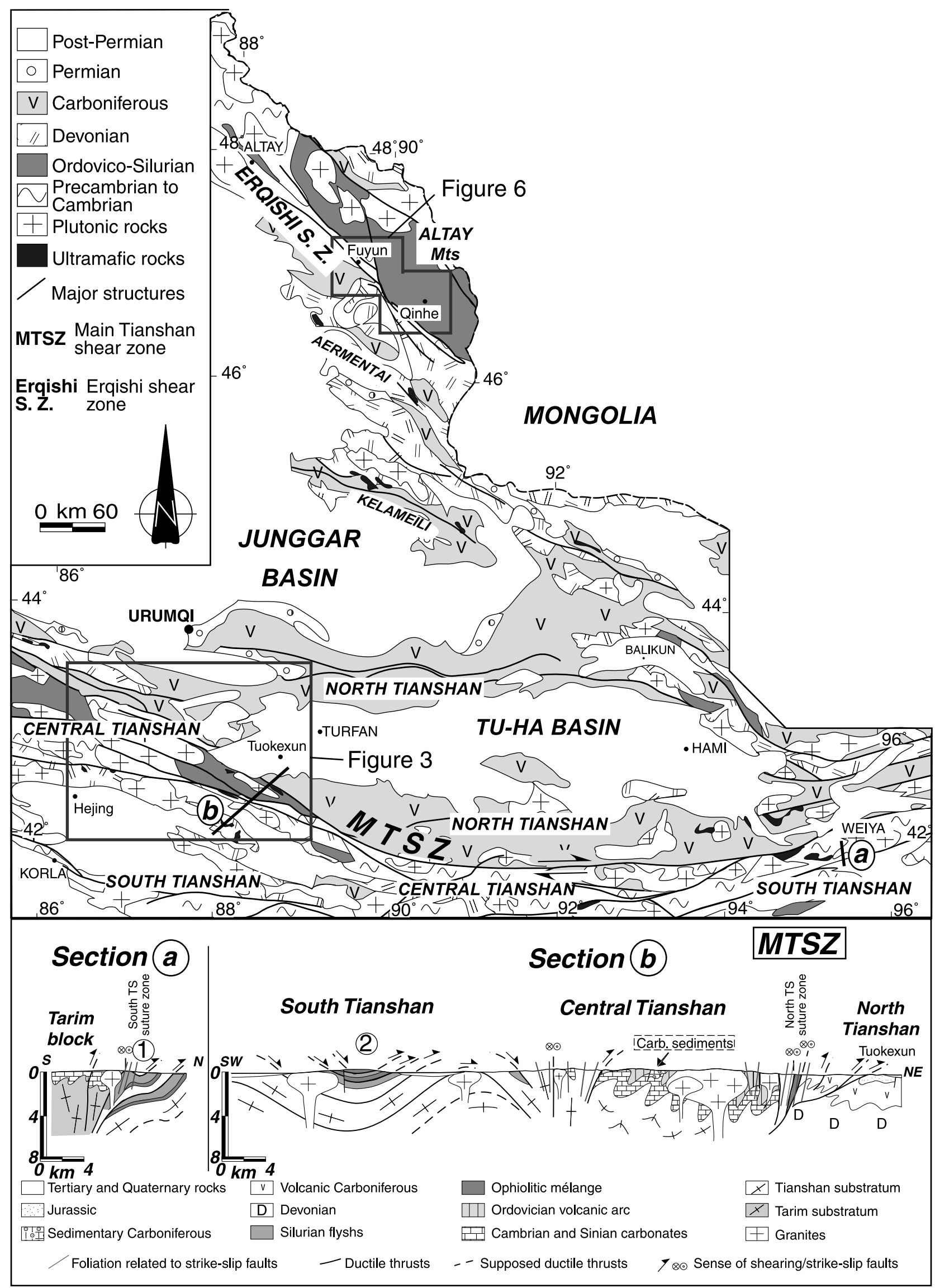


1994; Coleman, 1989] (Figure 1). The Tianshan orogenic belt separates the Siberian and Junggar microcontinents to the north from the Tarim and the Yili (which has a Tarimtype basement) blocks to the south and southwest [Ma et al., 1990; Xiao et al., 1990]. The structural development of the area and former Kazakhstan-Tianshan results from several tectonic events, mainly two subduction-collision stages: the first one during middle Paleozoic (1 on Figure 1) and the second one during late Paleozoic (2 on Figure 1) [Berzin et al., 1994; Burtman, 1975; Charvet et al., 2000; Coleman, 1989; Dobretsov et al., 1995; Gao et al., 1998; LaurentCharvet, 2001; Ma et al., 1990; Sengör et al., 1993; Shu et al., 1999b; Windley et al., 1990].

[7] The eastern and western Tianshan are commonly divided into three main units separated by the late Paleozoic strike-slip faults [Burtman, 1975; Coleman, 1989; Gao et al., 1998; Ma et al., 1993; Windley, 1990]. In this paper, we will focus on the eastern part of the Tianshan range. The southern unit and the South Tianshan, which extend all along the northern margin of the Tarim basin, are represented by Sinian marbles and limestones and Cambrian marbles, marls, and phosphatic rocks deposited on a Proterozoic gneissic substratum. The carbonates are interpreted as the continental margin sediments of the north passive margin of the Tarim block [Ma et al., 1993]. The Central Tianshan is mainly represented by Ordovician-Silurian volcanic rocks and Cambrian marbles on Proterozoic gneisses. In this unit, Lower Carboniferous conglomerates and limestones lie uncomformably on the oldest formations. During the middle Paleozoic the Tarim margin was accreted to the Ordovician continent-based volcanic arc of Central Tianshan along the Southern Tianshan Suture Zone (STSZ) (circled 1 on cross section a of Figure 2). Most of the models available in the literature [e.g., Allen et al., 1995; Burtman, 1975; Coleman, 1989; Gao et al., 1998; Windley, 1990] propose that the western part of the Tianshan suffered a main south verging deformation during the middle Paleozoic. Our recent tectonic and microtectonic observations [Laurent-Charvet, 2001] show that in the eastern part of the South and Central Tianshan units this accretion is characterized by northward thrusts and nappes of Proterozoic gneisses, Ordovician-Silurian sandstones, and volcanic and sedimentary Devonian rocks metamorphosed in greenschist facies. As shown by the cross sections a and b of Figure 2, the STSZ is underlined by an ophiolitic mélange with a schistosed metapelitic matrix containing Upper Silurian fossils [Ma et al., 1993]. Blocks of Sinian and Cambrian carbonates from the Tarim margin occur within this matrix. The mélange also includes various blocks of mafic to ultramafic rocks, diabases, Lower Devonian cherts [Gao et al., 1995], Tarim type marbles, and slabs of highpressure metamorphic rocks (circled 2 on cross section b of Figure 2) [Gao and Klemd, 2000; Gao et al., 1998; Shu et al., 1996]. Blueschist blocks were dated at 351 (muscovites associated to a retrogressive metamorphism in greenschists) and $415 \mathrm{Ma}$ (sodic amphiboles associated to a prograde metamorphism in blueschists) by ${ }^{40} \mathrm{Ar} /{ }^{39} \mathrm{Ar}$ method [Gao et al., 1995, 1998, 2000]. The suture zone is located between the north Tarim margin and the South Tianshan unit and can be observed in the Weiya area in eastern Tianshan (circled 1 on cross section a and map of Figure 2). This ophiolitic mélange also cropped out as nappes thrusted on Proterozoic gneisses in the Kumux area in the South Tianshan. Carboniferous granites and the coarse sandstones and conglomerates of Early Carboniferous age are sealing this Devonian deformation. The North Tianshan Suture Zone (NTSZ) marks the northern boundary of the Proterozoic basement of Ordovician volcanic arc (cross section b of Figure 2). In a few places the suture zone is underlaid by relics of an ophiolitic mélange with Ordovician mafic blocks [Che et al., 1994] and Cambro-Ordovician tuffs [Guo et al., 1993; Gao et al., 1998]. The Devonian north verging deformation also affected this suture zone and so constrains the age of this suture zone to the Middle to Late Devonian, like the STSZ. The NTSZ can be interpreted as the suture zone between the Central Tianshan volcanic arc and a northern continental microblock, which can be the basement of the North Tianshan unit (Figure 1).

[8] The limit between Central Tianshan and North Tianshan is represented by the Main Tianshan shear zone (MTSZ) (also locally named Aqqikkudug-Weiya shear zone [Shu et al., 1999a]) and is characterized by an east-west late Paleozoic dextral strike-slip motion [Laurent-Charvet et al., 2002]. The North Tianshan unit, whose substratum is not exposed and which surrounds the Late Permian Tu-Ha basin [Allen et al., 1995], is composed of two upper Paleozoic volcanic subunits: (1) a Lower to Middle Carboniferous calc-alkaline volcanic unit and (2) a Middle Devonian to Carboniferous calc-alkaline volcanic arc [Shu et al., 1999b, 2000]. This volcanic arc, called the Bogeda-Haerlike arc, is bounded by the Junggar block, which is limited to the north by the Devonian suture zone of Kelameili [Laurent-Charvet, 2001] (Figures 1 and 2). The North Tianshan unit suffered a Late Carboniferous north verging deformation in low temperature conditions. This last tangential deformation is sealed by the undeformed Permian conglomerates and molasses.

\subsection{Altay Mountains}

[9] To the northeast of the Kelameili suture zone (Figures 1 and 2), which limits the Junggar block, crop out several units representing the Mongolia-Siberia-type margin of the Eurasian block. It is made of a Proterozoic basement overlain by an early Paleozoic volcanosedimentary cover [ $L i$ and Bespaev, 1994]. These formations yielded some Silurian fossils belonging to the Tu-Wa fauna [Laurent-Charvet, 2001; Ma et al., 1993] and some Ordovician trilobites (e.g., Isotelus and Calyptaulax) [Laurent-Charvet, 2001], which confirm the Siberian origin of this region [LaurentCharvet, 2001; Zhou and Dean, 1996]. Late Paleozoic formations are of volcanic origin (rhyolites, basalts, and andesites). In spite of the presence of the fossils, large uncertainties still persist concerning the age of the different volcanic formations. As in western Junggar [Kwon et al., 1989], three successive stages of voluminous magmatism are recognized in the Chinese Altay region between the Late Caledonian and the Late Hercynian times [Liu, 1993]. A few 
granites, intruded in the Paleozoic cover, were dated by $\mathrm{Rb}$ $\mathrm{Sr}$ whole rock method and gave ages ranging from 390 to $180 \mathrm{Ma}$ with a main plutonic stage at $290-270 \mathrm{Ma}$ [Han et al., 1997, 1998; Liu, 1993].

\subsection{Transcurrent Deformations}

[10] After the earlier thrust tectonics in eastern and middle Tianshan orogen [Shu et al., 1997, 1998, 1999a], two main stages of transcurrent deformation were recognized in the Junggar area: first, a sinistral event in the spurs of Altay Mountains along the Erqishi-Irtysh shear zone [Allen et al., 1995; Dehandschutter et al., 1997; Melnikov et al., 1997, 1998] and then a widespread dextral event that reworked all preexisting structures all around the Junggar basin (e.g., the MTSZ). It was locally conjugated with sinistral ductile faults of limited amplitude in Central Tianshan [Laurent-Charvet et al., 2002]. These transcurrent regional motions are assumed to be responsible for the opening of several extensional basins such as the Junggar and Turfan ones [Allen et al., 1995].

\subsection{Tertiary Deformations}

[11] Finally, early to middle Miocene brittle structures developed in the whole area, as a consequence of the northward propagation of the Indo-Eurasia collision [Allen et al., 1999; Avouac et al., 1993; Hendrix et al., 1994]. It led to the intracontinental northward subduction of the Tarim block under the Tianshan Paleozoic orogen [Molnar and Tapponnier, 1975; Nishidai and Berry, 1991; Tapponnier and Molnar, 1979]. Today, the northward propagated deformation continues, and seismic activity is active in the whole Xinjiang province [Avouac and Tapponnier, 1993; Burchfiel et al., 1999; Cunningham et al., 1996; Nelson et al., 1987; Ritz et al., 1995].

\section{Strike-Slip Shear Zones in the Chinese Tianshan}

[12] The roughly east-west trending ductile shear zones in Tianshan are about $1 \mathrm{~km}$ wide and several hundreds of kilometers long. They separate the three main units constituting the Tianshan orogen (Figure 2). The offsets of these shear zones, and particularly that of the regional-scale Main Tianshan shear zone (MTSZ), are difficult to estimate because of the scale of structures and the lack of field markers preventing a precise mapping of geological structures before the strike-slip shearing. Nevertheless, owing to the regional scale of these structures it is likely that the horizontal displacement along these faults has been larger than tens of kilometers.

[13] Four main types of deformed rocks are exposed along the shear zones: Paleozoic mylonitic schists (flyschs and volcanic rocks), Proterozoic gneisses, folded migmatites, and mylonitic massive quartzites. All these lithologies display a conspicuous subhorizontal stretching lineation that trends systematically to a west-east direction. Associated to this stretching lineation, several types of microstructures can be observed such as shear bands and rigid porphyroclasts surrounded by microgranular dynamically recrystallized quartzofeldspathic aggregates. New structural and geochronological results providing constraints on the motion of the MTSZ are exposed below.

\subsection{Structural Study}

[14] On outcrops, mylonites from the MTSZ present a subhorizontal stretching lineation with an average direction of N110 in the western area (Figure 3 depiction 1) and N85 in the eastern part (Figure 3 depiction 2), carried by an E-W to ESE-WNW trending steeply dipping foliation. Kinematic criteria associated to the subhorizontal lineation include outcrop-scale plurimillimetric spaced shear bands, asymmetric plagioclase augens, boudinage, and asymmetric $\sigma$ type K-feldspar sigmoids with sericite and plagioclase recrystallized tails. All indicate a dextral sense of shearing. Many criteria in thin sections confirm this dextral strike-slip motion: "mica fish" structures, biotite-bearing shear bands (Figures $4 \mathrm{a}, 4 \mathrm{~b}$, and $4 \mathrm{~d}$ ), phenoclasts with recrystallized quartz and micas asymmetric tails (Figure 4c), $\sigma$-type porphyroclasts quartz, fibrous quartz, or chlorite asymmetric pressure shadows around rigid feldspar grains, etc. Dynamic recrystallization is also evident from quartz subgrains, grain boundary migration, grain nucleation, and quartz grains with strong undulose extinction and serrated and lobate boundaries. A study of quartz $c$ axis preferred orientation was carried out in mylonitic quartzites and orthogneisses that show such indications of dynamic recrystallization [Laurent-Charvet et al., 2002; Shu et al., 1999a]. Most patterns display a well-marked preferred orientation with a maximum located at the circumference (Figure 3). Maxima near $Z$ axis and basal $\langle a\rangle$ slip system activated during the noncoaxial deformation suggest that dextral shearing occurred under low temperature conditions $\left(<300^{\circ} \mathrm{C}\right)$ [Passchier and Trouw, 1996]. Pattern c shows two poles distant from the circumference, which indicates that rhomb $\langle a\rangle$ slip system was also activated. Those results are consistent with the various dextral kinematic criteria deduced from field and thin section observations all along the MTSZ. In Eastern Tianshan, evidences of an earlier sinistral deformation were found in a few samples: sinistral shear criteria and sinistral quartz $c$ axis preferred orientations with coeval activation of basal $\langle a\rangle$ and prismatic $\langle a\rangle$ slip systems, corresponding to medium-temperature conditions $\left(\sim 350^{\circ} \mathrm{C}\right)$. These sinistral shear indicators are partially overprinted by the subsequent dextral deformation [Laurent-Charvet et al., 2002] and may represent an earlier stage of transcurrent shearing along the eastern part of the MTSZ preceding the large-scale dextral motion.

\subsection{The ${ }^{40} \mathrm{Ar} /{ }^{39} \mathrm{Ar}$ Dating}

[15] Three places were sampled for ${ }^{40} \mathrm{Ar} /{ }^{39} \mathrm{Ar}$ dating in Chinese Tianshan (Figure 3): (1) the Mishigou area along the northern branch of the MTSZ, between Central Tianshan and North Tianshan (sample N3); (2) the Bindaban pass along the same northern MTSZ branch (sample TS07); (3) near Kumux along a southern branch of the MTSZ (sample TS520). The three selected samples are orthogneisses that were all dextrally deformed and contain well-preserved 


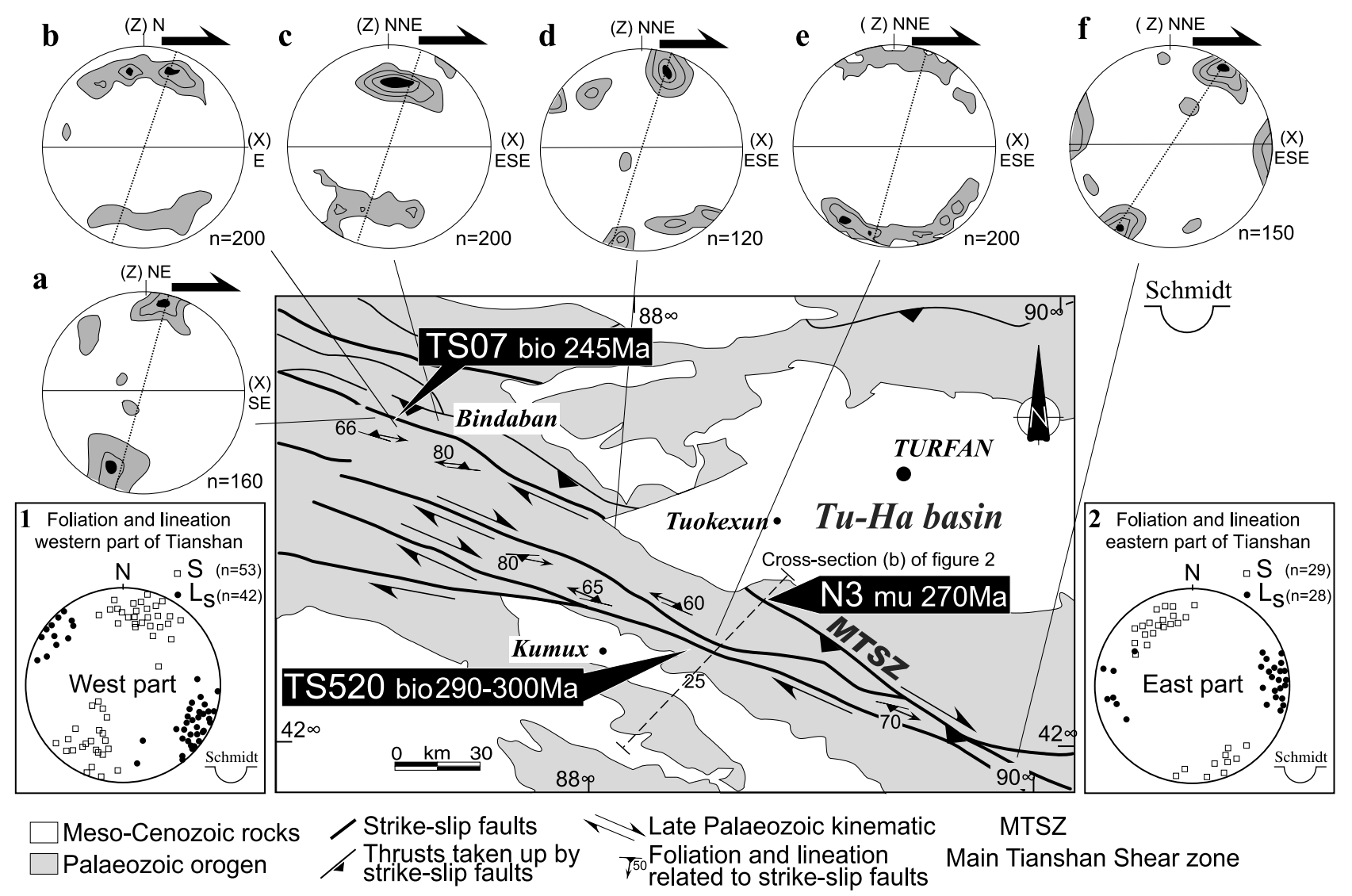

Figure 3. Structural synthetic map and $(a-f)$ stereograms of quartz $c$ axis fabrics and foliation and lineation (1 and 2) relative to ductile strike-slip faults in the central part of Tianshan (modified from Laurent-Charvet et al. [2002]). Location of dated samples is shown. See Figure 2 for location. Figure 3a shows contours 2 and 4 and $6 \%$ per $1 \%$ area. Figure $3 \mathrm{~b}$ shows contours 3, 5, and 7 and $9 \%$ per $1 \%$ area. Figure $3 \mathrm{c}$ shows contours 2, 4, and 6 and $8 \%$ per $1 \%$ area. Figure $3 \mathrm{~d}$ shows contours 2 and 4 and $6 \%$ per $1 \%$ area. Figure 3 e shows contours 3, 5, and 7 and $9 \%$ per $1 \%$ area. Figure 3 f shows contours 2 and 4 and $6 \%$ per $1 \%$ area.

biotites and muscovites. All the samples derive from former Proterozoic granites (dated at $1013 \pm 66 \mathrm{Ma}(\mathrm{U} / \mathrm{Pb}$ zircon age of $\mathrm{Hu}$ et al. [1999]) and at $826 \pm 30 \mathrm{Ma}(\mathrm{Rb} / \mathrm{Sr}$ whole rock age of Che et al. [1994])). In the Tianshan range, strike-slip ductile faulting has variably affected rocks of Proterozoic to Carboniferous ages. Details of our ${ }^{40} \mathrm{Ar} /{ }^{39} \mathrm{Ar}$ analytical procedure and locations of samples are given in Appendix A. Results are presented in Tables 1-3, and a summary of these results is given in Table 4 .

[16] Sample N3 is a muscovite-biotite gneiss for which biotite shows some evidence of chloritization, while muscovite remains unaltered. Like all deformed rocks along the MTSZ, the sample bears a vertical foliation associated with a subhorizontal stretching lineation and dextral shear criteria such as sigmoid feldspatic clasts with mica bearing tails. Step heating of a muscovite single grain done in Nanjing University [Shu et al., 1999a] yields a plateau date of $269.1 \pm 5.4 \mathrm{Ma}$ for $92 \%$ of the argon released (Table 2 and Figure 5a) [Shu et al., 1999a]. The atmospheric contamination was high in the very first steps and remains stable for the subsequent argon release.
[17] Sample TS07 from Bindaban pass (between Urumqi and Hejing) is a Proterozoic gneiss [Che et al., 1994]. The N100 foliation is steeply dipping to the north and also contains a horizontal stretching lineation. The sample is mainly composed of recrystallized quartz (with a LPO $\langle\mathrm{c}\rangle$ axis pattern compatible with a dextral deformation in low to medium-temperature conditions (Figure 3b)), plagioclase and perthite relicts, and micas parallel to the foliation. Shear bands with biotite, asymmetric dextral objects, and microfaulted feldspars also suggest the predominance of dextral shearing (Figure 4c). Twelve spot fusion laser probe experiments carried out on a biotite grain from this sample give data ranging between $237.2 \pm 3.7 \mathrm{Ma}$ and $267.6 \pm 6.4 \mathrm{Ma}$, with nine spots in the range $240-250 \mathrm{Ma}$ (Table 1 and Figure $5 \mathrm{~b}$ ). The three first experiments are characterized by a high atmospheric ${ }^{40} \mathrm{Ar}$ contamination ranging from $27 \%$ to $49 \%$ of ${ }^{40} \mathrm{Ar}$ (Table 1). This atmospheric argon probably represented a weakly bounded component to the lattice of the mica and was mainly released from the mineral surface and crystal defects. During these first experiments a mechanical disturbance of the mica surface has been 


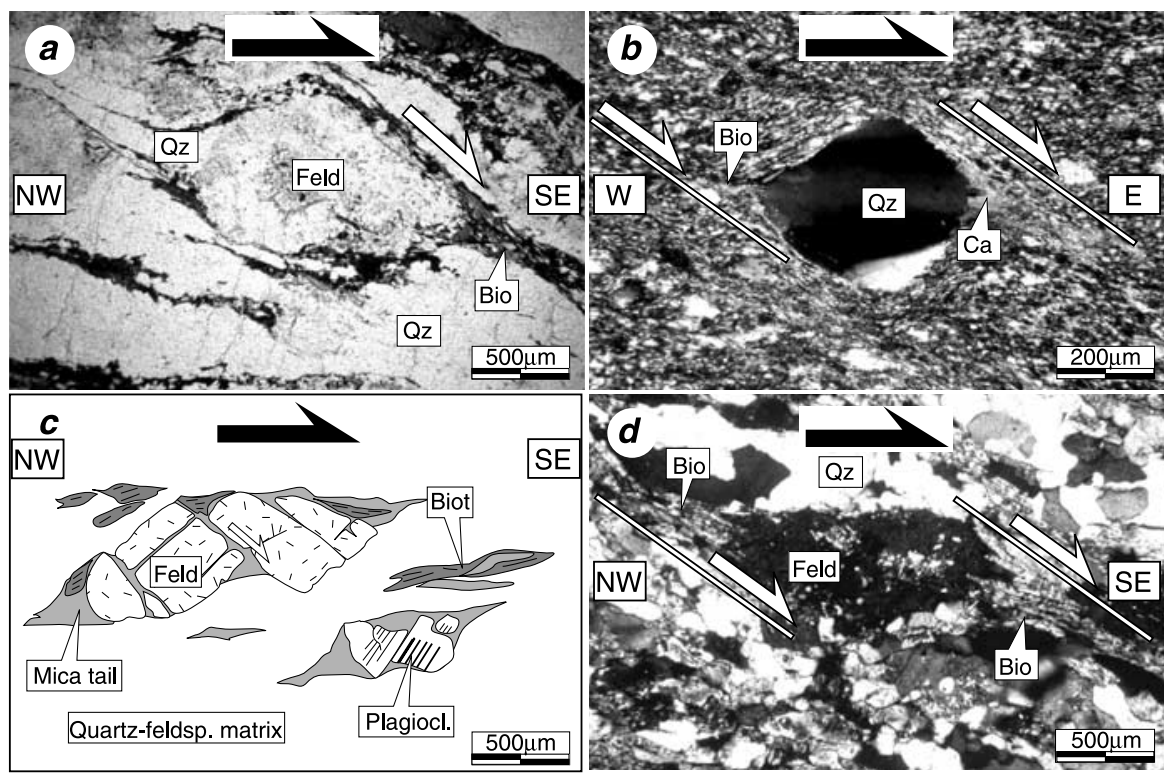

Figure 4. Examples of strike-slip microstructures along the main Tianshan shear zone. (a) Shear bands in Proterozoic orthogneiss in central Tianshan along the main ductile shear zone. Dextral sense of shear is indicated. (b) Shear bands and calcite tails around quartz phenoclast in Ordovician graywacke. Dextral sense of shearing in the eastern Tianshan is indicated. (c) Draw of a synthetical microfaulted feldspar and of an antithetical microfaulted plagioclase in an Ordovician graywacke. Dextral sense of shearing is indicated. (d) Biotite shear bands around a feldspatic clast in a Proterozoic orthogneiss along the Tianshan shear zone. Dextral sense of shearing is indicated. For whole pictures, foliation is parallel to large side. Abbreviations are as follows: Bio, biotite; $\mathrm{Ca}$, calcite; Feld, feldspar; and Qz, quartz.

observed through the camera placed above the sample in response to the strong energy supplied by the laser beam. It is suggested that it is the same atmospheric component that is present in the first gas fractions released in a step-heating experiment (see previous sample N3). For sample TS07 it must be noticed that the older apparent ages close to $265 \mathrm{Ma}$ are associated with the release of this weakly bounded argon, which could be due to a nonatmospheric composition of the initial argon. An isochron age of $244.7 \pm 2.6 \mathrm{Ma}$ in the ${ }^{36} \mathrm{Ar} /{ }^{40} \mathrm{Ar}$ versus ${ }^{39} \mathrm{Ar} /{ }^{40} \mathrm{Ar}$ isotope correlation plot with an initial ${ }^{40} \mathrm{Ar} /{ }^{36} \mathrm{Ar}$ ratio of 312.8.0 $\pm 15.5(\mathrm{MSWD}=3.06 \pm$ 0.45 ) has been calculated with the least discordant spots from this sample (Table 4).

[18] Along the southern branch of the MTSZ, in the area of Kumux, deformed rocks with dextral structures, similar to those described above but not necessarily contemporaneous, are mainly gneisses and mylonites, which outcrop along the shear zone. Sample TS520 is an orthogneiss in which only one family of biotite can be detected on textural grounds in coexistence with actinolite and muscovite formed during a high to medium-temperature event. Biotite is parallel to the foliation and to shear bands and remains unaffected by weathering (Figure 4d). Twelve spots obtained on a single mica grain have ages ranging from $274.8 \pm 5.3 \mathrm{Ma}$ to $302.0 \pm 4.5 \mathrm{Ma}$ (Figure 5c). The spots, which are in line along the length of the crystal, show that this scattering does not correspond to any systematic core versus rim distribution of apparent ages (Table 1 and Figure $5 \mathrm{c}$ ). As for the previous sample, the atmospheric contamination is significantly higher in the first spot. An intercept age of $292.6 \pm 3.2 \mathrm{Ma}$ (Figure 5c and Table 4) has been obtained in the ${ }^{36} \mathrm{Ar} /{ }^{40} \mathrm{Ar}$ versus ${ }^{39} \mathrm{Ar} /{ }^{40} \mathrm{Ar}$ isotope correlation plot with an initial ${ }^{40} \mathrm{Ar} /{ }^{36} \mathrm{Ar}$ ratio of $252.4 \pm$ 106.8 and $\mathrm{a}$ MSWD $=2.54 \pm 0.53$.

[19] In western Central Tianshan a ${ }^{40} \mathrm{Ar} /{ }^{39} \mathrm{Ar}$ plateau age of $250.5 \pm 0.3 \mathrm{Ma}$ has been reported on biotite from a similar mylonitic gneiss [Chen et al., 1999]. Laser probe ages of 240-250 Ma obtained on biotite TS07 during this study are in a similar range. According to Harrison et al. [1985] these ages can be interpreted to record cooling at about $300^{\circ}-350^{\circ} \mathrm{C}$ and coeval closure of the biotite chronometer. Analysis of quartz $c$ axis fabrics indicates that the last increments of dextral deformation along the MTSZ occurred nearly within the same temperature range $\left(300^{\circ}-\right.$ $350^{\circ} \mathrm{C}$ ). This suggests that biotite probably started to retain argon immediately after this last deformation and coeval recrystallization. Therefore it is very likely that dextral motion along the northern branch of the MTSZ from which sample TS07 originates ended in the Late Permian accommodating the final exhumation of metamorphic rocks. By contrast, sample TS520 from a southern branch of the MTSZ yields biotite Upper Carboniferous ages (290-300 Ma), which suggest that this branch escaped the last episode of Permian dextral shearing and low-temperature recrystallization. However, and as noticed above, dextral shearing also predominates within sample TS520. However, the metamorphic conditions associated with this shearing are of higher temperature than along the northern branch of the 
Table 1. The ${ }^{40} \mathrm{Ar} /{ }^{39} \mathrm{Ar}$ Age Data Resulting From Laser Probe Fusion Experiments of Single Grains Extracted From the Tianshan Area (TS07 and TS520), Qinhe Area (TS330), and Fuyun Metamorphic Zone (TS247, TS305, and TS310)

\begin{tabular}{|c|c|c|c|c|c|c|}
\hline & ${ }^{40} \mathrm{Ar} *{ }^{39} \mathrm{Ar}$ & ${ }^{36} \mathrm{Ar}^{40} \mathrm{Ar}^{\mathrm{a}}$ & ${ }^{39} \mathrm{Ar} /{ }^{40} \mathrm{Ar}$ & ${ }^{37} \mathrm{Ar} /{ }^{39} \mathrm{Ar}$ & Atm, \% & Age $\pm 1 \sigma$ \\
\hline \multicolumn{7}{|c|}{ TSO7 Biotite $J=0.015627$} \\
\hline 1 & 9.094 & 1.652 & 0.0562 & 0.06 & 48.8 & $239.7 \pm 6.4$ \\
\hline 2 & 9.977 & 1.038 & 0.0694 & 0.0 & 30.6 & $261.4 \pm 6.4$ \\
\hline 3 & 10.231 & 0.917 & 0.0712 & 0.0 & 27.1 & $267.6 \pm 6.4$ \\
\hline 4 & 9.375 & 0.294 & 0.0973 & 0.0 & 8.7 & $246.7 \pm 2.3$ \\
\hline 5 & 9.649 & 0.269 & 0.0952 & 0.0 & 7.9 & $253.4 \pm 3.5$ \\
\hline 6 & 9.425 & 0.287 & 0.097 & 0.0 & 8.5 & $247.9 \pm 4.2$ \\
\hline 7 & 9.321 & 0.278 & 0.0983 & 0.0 & 8.2 & $245.4 \pm 3.9$ \\
\hline 8 & 8.993 & 0.323 & 0.1005 & 0.0 & 9.5 & $237.2 \pm 3.7$ \\
\hline 9 & 9.229 & 0.204 & 0.1017 & 0.006 & 6,0 & $243.1 \pm 2.0$ \\
\hline 10 & 9.523 & 0.404 & 0.0924 & 0.0 & 11.9 & $250.3 \pm 4.0$ \\
\hline 11 & 9.271 & 0.14 & 0.1033 & 0.016 & 4.1 & $244.1 \pm 5.2$ \\
\hline 12 & 9.17 & 0.205 & 0.1024 & 0.0 & 6.0 & $241.6 \pm 3.4$ \\
\hline \multicolumn{7}{|c|}{ TS520 Biotite $J=0.015627$} \\
\hline 1 & 11.09 & 1.124 & 0.0602 & 0.135 & 33.2 & $288.4 \pm 11.1$ \\
\hline 2 & 10.528 & 0.214 & 0.0889 & 0.121 & 6.3 & $274.8 \pm 5.3$ \\
\hline 3 & 11.661 & 0.095 & 0.0833 & 0.0 & 2.8 & $302.0 \pm 4.3$ \\
\hline 4 & 10.84 & 0.197 & 0.0868 & 0.039 & 5.8 & $282.3 \pm 5.5$ \\
\hline 5 & 11.064 & 0.223 & 0.0844 & 0.039 & 6.6 & $287.7 \pm 2.7$ \\
\hline 6 & 11.235 & 0.095 & 0.0864 & 0.0 & 2.8 & $291.8 \pm 3.9$ \\
\hline 7 & 11.561 & 0.013 & 0.0861 & 0.0 & 0.3 & $299.6 \pm 5.3$ \\
\hline 8 & 11.182 & 0.099 & 0.0868 & 0.013 & 2.9 & $290.5 \pm 3.3$ \\
\hline 9 & 11.009 & 0.172 & 0.0862 & 0.0 & 5,0 & $286.4 \pm 2.4$ \\
\hline 10 & 11.222 & 0.197 & 0.0839 & 0.0 & 5.8 & $291.5 \pm 4.6$ \\
\hline 11 & 11.368 & 0.032 & 0.0871 & 0.037 & 0.9 & $295.0 \pm 3.6$ \\
\hline 12 & 10.743 & 0.232 & 0.0866 & 0.118 & 6.8 & $280.0 \pm 3.7$ \\
\hline \multicolumn{7}{|c|}{ TS330 Biotite $J=0.015627$} \\
\hline 1 & 10.27 & 1.450 & 0.0556 & 0.0 & 42.8 & $268.5 \pm 5.8$ \\
\hline 2 & 8.743 & 1.038 & 0.0792 & 0.0 & 30.6 & $231.1 \pm 2.5$ \\
\hline 3 & 10.499 & 0.691 & 0.0757 & 0.017 & 20.4 & $274.1 \pm 5.0$ \\
\hline 4 & 9.331 & 0.626 & 0.0873 & 0.0 & 18.5 & $245.6 \pm 4.1$ \\
\hline 5 & 10,000 & 0.360 & 0.0893 & 0.045 & 10.6 & $262.0 \pm 2.6$ \\
\hline 6 & 9.553 & 0.192 & 0.0987 & 0.0 & 5.6 & $251.0 \pm 3.2$ \\
\hline 7 & 10.128 & 0.583 & 0.0817 & 0.0 & 17.2 & $265.1 \pm 2.6$ \\
\hline 8 & 10.574 & 0.171 & 0.0897 & 0.01 & 5.0 & $275.9 \pm 3.4$ \\
\hline 9 & 9.100 & 0.280 & 0.1007 & 0.0 & 8.2 & $239.9 \pm 5.8$ \\
\hline 10 & 9.775 & 0.151 & 0.0977 & 0.0 & 4.4 & $256.5 \pm 2.9$ \\
\hline 11 & 9.837 & 0.202 & 0.0955 & 0.012 & 5.9 & $258.0 \pm 2.2$ \\
\hline 12 & 10.041 & 0.247 & 0.0923 & 0.033 & 7.3 & $263.0 \pm 2.1$ \\
\hline 13 & 10.013 & 0.225 & 0.0932 & 0.02 & 6.6 & $262.3 \pm 1.3$ \\
\hline 14 & 9.643 & 0.272 & 0.0952 & 0.02 & 8.0 & $253.2 \pm 2.4$ \\
\hline \multicolumn{7}{|c|}{ TS247 Biotite $J=0.015627$} \\
\hline 1 & 10.783 & 0.955 & 0.0665 & 0.0 & 28.2 & $280.9 \pm 5.7$ \\
\hline 2 & 10.468 & 0.438 & 0.0831 & 0.0 & 12.9 & $273.3 \pm 8.3$ \\
\hline 3 & 10.673 & 0.586 & 0.0774 & 0.0 & 17.3 & $278.3 \pm 3.3$ \\
\hline 4 & 9.814 & 0.631 & 0.0828 & 0.047 & 18.6 & $257.4 \pm 5.4$ \\
\hline 5 & 10.206 & 0.238 & 0.091 & 0.014 & 7.0 & $267.0 \pm 1.9$ \\
\hline 6 & 10.349 & 1.76 & 0.0463 & 0.083 & 52.0 & $270.5 \pm 9.7$ \\
\hline 7 & 9.854 & 0.376 & 0.0901 & 0.016 & 11.1 & $258.4 \pm 4.0$ \\
\hline 8 & 10.36 & 2.056 & 0.0378 & 0.057 & 60.7 & $270.7 \pm 8.8$ \\
\hline 9 & 10.8 & 1.351 & 0.0556 & 0.14 & 39.9 & $281.4 \pm 8.0$ \\
\hline 10 & 9.865 & 0.288 & 0.0927 & 0.034 & 8.5 & $258.7 \pm 4.5$ \\
\hline 11 & 10.154 & 0.692 & 0.0783 & 0.027 & 20.4 & $265.7 \pm 3.1$ \\
\hline 12 & 10.193 & 0.2 & 0.0921 & 0.0 & 5.9 & $266.7 \pm 1.6$ \\
\hline 13 & 10.177 & 0.189 & 0.0927 & 0.0 & 5.6 & $266.3 \pm 3.0$ \\
\hline 14 & 10.061 & 0.209 & 0.0932 & 0.0 & 6.2 & $263.4 \pm 2.1$ \\
\hline 15 & 10.356 & 0.143 & 0.0924 & 0.0 & 4.2 & $270.6 \pm 4.5$ \\
\hline 16 & 10.188 & 0.254 & 0.0907 & 0.0 & 7.5 & $266.5 \pm 3.2$ \\
\hline \multicolumn{7}{|c|}{ TS305 Biotite $J=0.015627$} \\
\hline 1 & 8.774 & 2.646 & 0.0247 & 0.033 & 78.1 & $231.8 \pm 16.6$ \\
\hline 2 & 9.413 & 1.062 & 0.0727 & 0.052 & 31.3 & $247.6 \pm 4.6$ \\
\hline 3 & 9.62 & 0.204 & 0.0976 & 0.0 & 6.0 & $252.7 \pm 5.1$ \\
\hline 4 & 9.922 & 0.141 & 0.0965 & 0.019 & 4.1 & $260.1 \pm 9.6$ \\
\hline
\end{tabular}


Table 1. (continued)

\begin{tabular}{|c|c|c|c|c|c|c|}
\hline & ${ }^{40} \mathrm{Ar} * /{ }^{39} \mathrm{Ar}$ & ${ }^{36} \mathrm{Ar} /{ }^{40} \mathrm{Ar}^{\mathrm{a}}$ & ${ }^{39} \mathrm{Ar} /{ }^{40} \mathrm{Ar}$ & ${ }^{37} \mathrm{Ar} /{ }^{39} \mathrm{Ar}$ & Atm, $\%$ & Age $\pm 1 \sigma$ \\
\hline 5 & 9.201 & 0.217 & 0.1016 & 0.0 & 6.4 & $242.4 \pm 7.2$ \\
\hline 6 & 9.321 & 0.232 & 0.0998 & 0.0 & 6.8 & $245.3 \pm 5.5$ \\
\hline 7 & 8.727 & 0.413 & 0.1005 & 0.008 & 12.2 & $230.7 \pm 5.4$ \\
\hline 8 & 8.998 & 0.478 & 0.0954 & 0.0 & 14.1 & $237.4 \pm 6.6$ \\
\hline 9 & 9.274 & 0.545 & 0.0903 & 0.0 & 16.1 & $244.2 \pm 4.0$ \\
\hline 10 & 9.385 & 0.282 & 0.0976 & 0.018 & 8.3 & $246.9 \pm 4.7$ \\
\hline 11 & 9.259 & 0.247 & 0.1000 & 0.02 & 7.3 & $243.8 \pm 3.0$ \\
\hline 12 & 9.387 & 0.173 & 0.1009 & 0.005 & 5.1 & $247.0 \pm 4.0$ \\
\hline 13 & 9.406 & 0.299 & 0.0968 & 0.026 & 8.8 & $247.4 \pm 5.6$ \\
\hline \multicolumn{7}{|c|}{ TS310 Biotite $1 \mathrm{~J}=0.015627$} \\
\hline 1 & 6.776 & 3.247 & 0.0059 & 0.179 & 95.9 & $181.6 \pm 63.7$ \\
\hline 2 & 5.427 & 2.891 & 0.0268 & 0.0 & 85.4 & $146.9 \pm 22.1$ \\
\hline 3 & 8.304 & 1.836 & 0.0550 & 0.233 & 54.2 & $220.1 \pm 12.7$ \\
\hline 4 & 5.255 & 3.030 & 0.0199 & 0.101 & 89.5 & $142.4 \pm 27.8$ \\
\hline 5 & 8.614 & 2.351 & 0.0354 & 0.024 & 69.4 & $227.8 \pm 11.4$ \\
\hline 6 & 8.618 & 2.331 & 0.0359 & 0.0 & 68.8 & $228.0 \pm 10.0$ \\
\hline 7 & 7.535 & 2.032 & 0.0529 & 0.014 & 60,0 & $200.8 \pm 11.5$ \\
\hline \multicolumn{7}{|c|}{ TS310 Biotite $2 J=0.015627$} \\
\hline 1 & 8.045 & 2.164 & 0.0447 & 0.024 & 63.9 & $213.6 \pm 10.3$ \\
\hline 2 & 7.441 & 2.182 & 0.0477 & 0.0 & 64.4 & $198.5 \pm 22.8$ \\
\hline 3 & 8.977 & 1.461 & 0.0632 & 0.155 & 43.1 & $236.8 \pm 5.1$ \\
\hline 4 & 12.213 & 1.437 & 0.0471 & 0.0 & 42.4 & $315.1 \pm 24.2$ \\
\hline 5 & 9.533 & 0.488 & 0.0897 & 0.004 & 14.4 & $250.5 \pm 7.7$ \\
\hline 6 & 9.317 & 0.281 & 0.0983 & 0.204 & 8.3 & $245.2 \pm 1.9$ \\
\hline 7 & 8.943 & 0.763 & 0.0864 & 0.128 & 22.5 & $236 \pm 2.6$ \\
\hline 8 & 9.246 & 0.423 & 0.0946 & 0.076 & 12.5 & $243.5 \pm 4.9$ \\
\hline
\end{tabular}

${ }^{\mathrm{a}}$ Multiply by 1000 .

Table 2. The ${ }^{40} \mathrm{Ar} /{ }^{39} \mathrm{Ar}$ Age Data Resulting From Step-Heating Experiments on Muscovite Separated From the Tianshan Shear Zone (N3) and the Fuyun Area (TS330)

\begin{tabular}{|c|c|c|c|c|c|c|c|}
\hline Step & ${ }^{40} \mathrm{Ar}, \%$ & ${ }^{36} \mathrm{Ar} /{ }^{40} \mathrm{Ar}^{\mathrm{b}}$ & ${ }^{39} \mathrm{Ar} /{ }^{36} \mathrm{Ar}$ & ${ }^{37} \mathrm{Ar} /{ }^{39} \mathrm{Ar}$ & ${ }^{39} \mathrm{Ar}, \%$ & Ar Ratio, $\%$ & Age $\pm 1 \sigma$ \\
\hline \multicolumn{8}{|c|}{ N3 Muscovite $J=0.02635764$} \\
\hline 1 & 0.02 & 3.282 & 1.47 & 4.672 & 0.02 & 3.02 & $276.2 \pm 4.5$ \\
\hline 2 & 0.23 & 2.277 & 27.53 & 0.027 & 0.27 & 32.71 & $232.6 \pm 4.2$ \\
\hline 3 & 1.16 & 0.973 & 136.17 & 0.051 & 1.31 & 71.17 & $239.2 \pm 4.2$ \\
\hline 4 & 4.47 & 0.422 & 310.91 & 1.784 & 4.09 & 87.43 & $292.3 \pm 4.3$ \\
\hline 5 & 54.37 & 0.038 & 4296.25 & 0.009 & 54.18 & 98.75 & $269.9 \pm 4.2$ \\
\hline 6 & 7.6 & 0.088 & 1888.29 & 0.025 & 7.91 & 97.26 & $259.2 \pm 4.2$ \\
\hline 7 & 12.54 & 0.065 & 2505.07 & 0.076 & 12.78 & 97.93 & $264.4 \pm 4.2$ \\
\hline 8 & 18.31 & 0.058 & 2747.51 & 0.53 & 18.12 & 98.15 & $271.8 \pm 4.2$ \\
\hline 9 & 1.31 & 0.722 & 179.41 & 0.046 & 1.31 & 78.57 & $267.8 \pm 4.2$ \\
\hline Step & ${ }^{40} \mathrm{Ar} * 39 \mathrm{Ar}$ & ${ }^{36} \mathrm{Ar} /{ }^{40} \mathrm{Ar}^{\mathrm{b}}$ & ${ }^{39} \mathrm{Ar} /{ }^{40} \mathrm{Ar}^{\mathrm{b}}$ & ${ }^{37} \mathrm{Ar} /{ }^{39} \mathrm{Ar}$ & ${ }^{39} \mathrm{Ar}, \%$ & Atm, $\%$ & Age $\pm 1 \sigma$ \\
\hline \multicolumn{8}{|c|}{ TS330 Muscovite $J=0.015627$} \\
\hline 1 & 2.901 & 2.698 & 0.0697 & 0.005 & 0.0 & 79.7 & $80.0 \pm 58.7$ \\
\hline 2 & 7.473 & 2.027 & 0.0536 & 0.000 & 0.2 & 59.9 & $199.3 \pm 17.5$ \\
\hline 3 & 9.966 & 0.384 & 0.0889 & 0.089 & 0.4 & 11.3 & $261.1 \pm 8.4$ \\
\hline 4 & 9.737 & 0.311 & 0.0932 & 0.007 & 0.5 & 9.2 & $255.5 \pm 6.2$ \\
\hline 5 & 9.421 & 0.223 & 0.0991 & 0.026 & 0.5 & 6.6 & $247.8 \pm 5.1$ \\
\hline 6 & 9.908 & 0.159 & 0.0961 & 0.000 & 1.3 & 4.7 & $259.7 \pm 3.8$ \\
\hline 7 & 9.548 & 0.031 & 0.1037 & 0.000 & 26.5 & 0.9 & $250.9 \pm 1.5$ \\
\hline 8 & 9.514 & 0.024 & 0.1043 & 0.001 & 12.5 & 0.7 & $250.1 \pm 1.2$ \\
\hline 9 & 9.41 & 0.037 & 0.105 & 0.003 & 12.9 & 1.1 & $247.5 \pm 1.8$ \\
\hline 10 & 9.447 & 0.075 & 0.1034 & 0.000 & 1.9 & 2.2 & $248.4 \pm 3.6$ \\
\hline 11 & 9.481 & 0.105 & 0.1021 & 0.009 & 1.2 & 3.1 & $249.3 \pm 3.8$ \\
\hline 12 & 9.704 & 0.084 & 0.1004 & 0.013 & 1.4 & 2.4 & $254.7 \pm 5.3$ \\
\hline 13 & 9.521 & 0.038 & 0.1038 & 0.01 & 11.9 & 1.1 & $250.2 \pm 1.1$ \\
\hline 14 & 9.379 & 0.037 & 0.1054 & 0.016 & 4.3 & 1.1 & $246.8 \pm 1.5$ \\
\hline 15 & 9.51 & 0.008 & 0.1048 & 0.004 & 24.5 & 0.2 & $250.0 \pm 1.3$ \\
\hline
\end{tabular}

${ }^{a} \mathrm{~N} 3$ dated at Nanjing University; TS330 dated at Montpellier University.

${ }^{\mathrm{b}}$ Multiply by 1000 . 
Table 3. The ${ }^{40} \mathrm{Ar} /{ }^{39} \mathrm{Ar}$ Age Data Resulting From Laser Probe Fusion Experiments on Polished Sections From Amphibolites of the Fuyun Metamorphic Zone (TS294 and TS295)

\begin{tabular}{|c|c|c|c|c|c|c|}
\hline & ${ }^{40} \mathrm{Ar} *{ }^{39} \mathrm{Ar}$ & ${ }^{36} \mathrm{Ar}^{40}{ }^{40} \mathrm{rr}^{\mathrm{a}}$ & ${ }^{39} \mathrm{Ar} r{ }^{40} \mathrm{Ar}$ & ${ }^{37} \mathrm{Ar} /{ }^{39} \mathrm{Ar}$ & Atm, $\%$ & Age $\pm 1 \sigma$ \\
\hline \multicolumn{7}{|c|}{ TS294 Amphiboles $J=0.015627$} \\
\hline 1 & 9.748 & 0.681 & 0.0819 & 15.681 & 20.1 & $255.8 \pm 9.9$ \\
\hline 2 & 7.334 & 1.031 & 0.0947 & 11.084 & 30.4 & $195.8 \pm 6.8$ \\
\hline 3 & 9.764 & 0.669 & 0.082 & 12.185 & 19.7 & $256.2 \pm 6.5$ \\
\hline 4 & 9.351 & 0.802 & 0.0815 & 13.223 & 23.7 & $246.1 \pm 9.1$ \\
\hline 5 & 9.377 & 0.973 & 0.0759 & 14.481 & 28.7 & $246.7 \pm 8.6$ \\
\hline 6 & 8.378 & 1.501 & 0.0663 & 11.898 & 44.3 & $222.0 \pm 8.5$ \\
\hline 7 & 9.043 & 0.904 & 0.081 & 13.82 & 26.7 & $238.5 \pm 11.7$ \\
\hline 8 & 9.101 & 0.657 & 0.0885 & 10.552 & 19.4 & $239.9 \pm 3.4$ \\
\hline 9 & 8.775 & 0.834 & 0.0858 & 10.589 & 24.6 & $231.8 \pm 7.1$ \\
\hline 10 & 10.114 & 0.758 & 0.0767 & 14.55 & 22.3 & $264.7 \pm 5.6$ \\
\hline 11 & 8.758 & 0.854 & 0.0853 & 9.282 & 25.2 & $231.4 \pm 3.9$ \\
\hline 12 & 10.731 & 0.617 & 0.0761 & 15.194 & 18.2 & $279.7 \pm 12.8$ \\
\hline \multicolumn{7}{|c|}{ TS295 Amphiboles $J=0.015627$} \\
\hline 1 & 13.431 & 2.28 & 0.0242 & 17.791 & 67.3 & $343.7 \pm 26.1$ \\
\hline 2 & 12.747 & 2.331 & 0.0243 & 21.776 & 68.9 & $327.7 \pm 89.5$ \\
\hline 3 & 7.873 & 2.569 & 0.0305 & 18.133 & 75.9 & $209.3 \pm 20.1$ \\
\hline 4 & 12.329 & 1.732 & 0.0394 & 19.997 & 51.2 & $317.9 \pm 48.5$ \\
\hline 5 & 10.175 & 2.386 & 0.0289 & 14.825 & 70.5 & $266.2 \pm 25.0$ \\
\hline 6 & 5.18 & 2.412 & 0.0553 & 13.031 & 71.2 & $140.4 \pm 36.1$ \\
\hline 7 & 13.894 & 1.348 & 0.0432 & 14.685 & 39.8 & $354.5 \pm 55.3$ \\
\hline 8 & 9.297 & 2.265 & 0.0355 & 15.897 & 66.9 & $244.7 \pm 13.4$ \\
\hline 9 & 10.867 & 1.944 & 0.0391 & 14.421 & 57.4 & $283.0 \pm 14.8$ \\
\hline 10 & 6.847 & 2.90 & 0.0208 & 15.871 & 85.7 & $183.4 \pm 21.5$ \\
\hline 11 & 9.90 & 1.426 & 0.0584 & 12.724 & 42.1 & $259.5 \pm 12.1$ \\
\hline 12 & 8.453 & 1.854 & 0.0534 & 13.779 & 54.7 & $223.9 \pm 12.8$ \\
\hline 13 & 8.559 & 1.636 & 0.0603 & 12.599 & 48.3 & $226.5 \pm 7.3$ \\
\hline 14 & 9.193 & 1.741 & 0.0527 & 11.142 & 51.4 & $242.2 \pm 11.1$ \\
\hline 15 & 9.487 & 1.445 & 0.0603 & 12.879 & 42.7 & $249.4 \pm 13.7$ \\
\hline
\end{tabular}

${ }^{\mathrm{a}}$ Multiply by 1000 .

MTSZ. Therefore biotite ages of 290-300 Ma most probably represent the time when the rocks crossed the $300^{\circ}-$ $350^{\circ} \mathrm{C}$ isotherm, some millions years after the first stage of dextral shearing. This indicates that within a relatively restricted area (Figure 3), dextral movements recorded by parallel NW-SE shear zones are not synchronous. Moreover, structural studies carried out in eastern Tianshan reveal relics of a former sinistral strike-slip shearing in mediumto high-temperature conditions [Laurent-Charvet et al., 2002] similar to those recorded by the sample TS520. Thus ages of 290-300 Ma could place a minimum age constraints for an earlier strike-slip motion mainly preserved in the southern branch of the MTSZ. A sinistral motion could occur before this event in the eastern Tianshan. We do not want to infer that this earlier sinistral motion in the eastern MTSZ was synchronous with the dextral one recorded by sample TS520 in the central part (dated at $290 \mathrm{Ma}$ ).

[20] Along the northern branch of the MTSZ the meaning of the $268.8 \pm 5.4 \mathrm{Ma}$ age on a neoformed muscovite N3 from a dextral shear zone remains uncertain compared to the biotite Permian ages. Because of a higher closure temperature for argon in muscovite than in biotite $\left(50^{\circ}-100^{\circ}\right.$ higher depending on the authors), a possibility is that this age reflects an early stage of cooling during dextral shearing, therefore suggesting that this shearing lasted over at least $20 \mathrm{Ma}$. Alternatively, owing to the few heating steps performed, and because the step-heating procedure tends to blur isotopic gradients in micas in response to their deshy-

Table 4. The ${ }^{40} \mathrm{Ar} /{ }^{39} \mathrm{Ar}$ Retained Ages and Used Method for Each

\begin{tabular}{|c|c|c|c|c|}
\hline Samples & Area & Mineral & Method & Retained Age \\
\hline N3 & northern branch of MTSZ & muscovite & step heating (plateau) & $269.1 \pm 5.4$ \\
\hline TS07 & northern branch of MTSZ & biotite & spot fusion (isochron) & $244.7 \pm 2.6$ \\
\hline TS520 & southern branch of MTSZ & biotite & spot fusion (isochron) & $292.6 \pm 3.2$ \\
\hline \multirow[t]{2}{*}{ TS330 } & Qinhe area & biotite & spot fusion (isochron) & $261.4 \pm 3.2$ \\
\hline & & muscovite & step heating (plateau) & $249.9 \pm 2.2$ \\
\hline TS247 & Fuyun complex & biotite & spot fusion (isochron) & $265.6 \pm 2.5$ \\
\hline TS305 & Fuyun complex & biotite & spot fusion (isochron) & $245.4 \pm 2.5$ \\
\hline TS310 & Fuyun complex & biotites & spot fusion (isochron) & $244.0 \pm 2.6$ \\
\hline TS294 & Fuyun complex & amphiboles & spot fusion (isochron) on polished sections & $244.1 \pm 2.8$ \\
\hline TS295 & Fuyun complex & amphiboles & spot fusion (isochron) on polished sections & $249.1 \pm 3.8$ \\
\hline
\end{tabular}



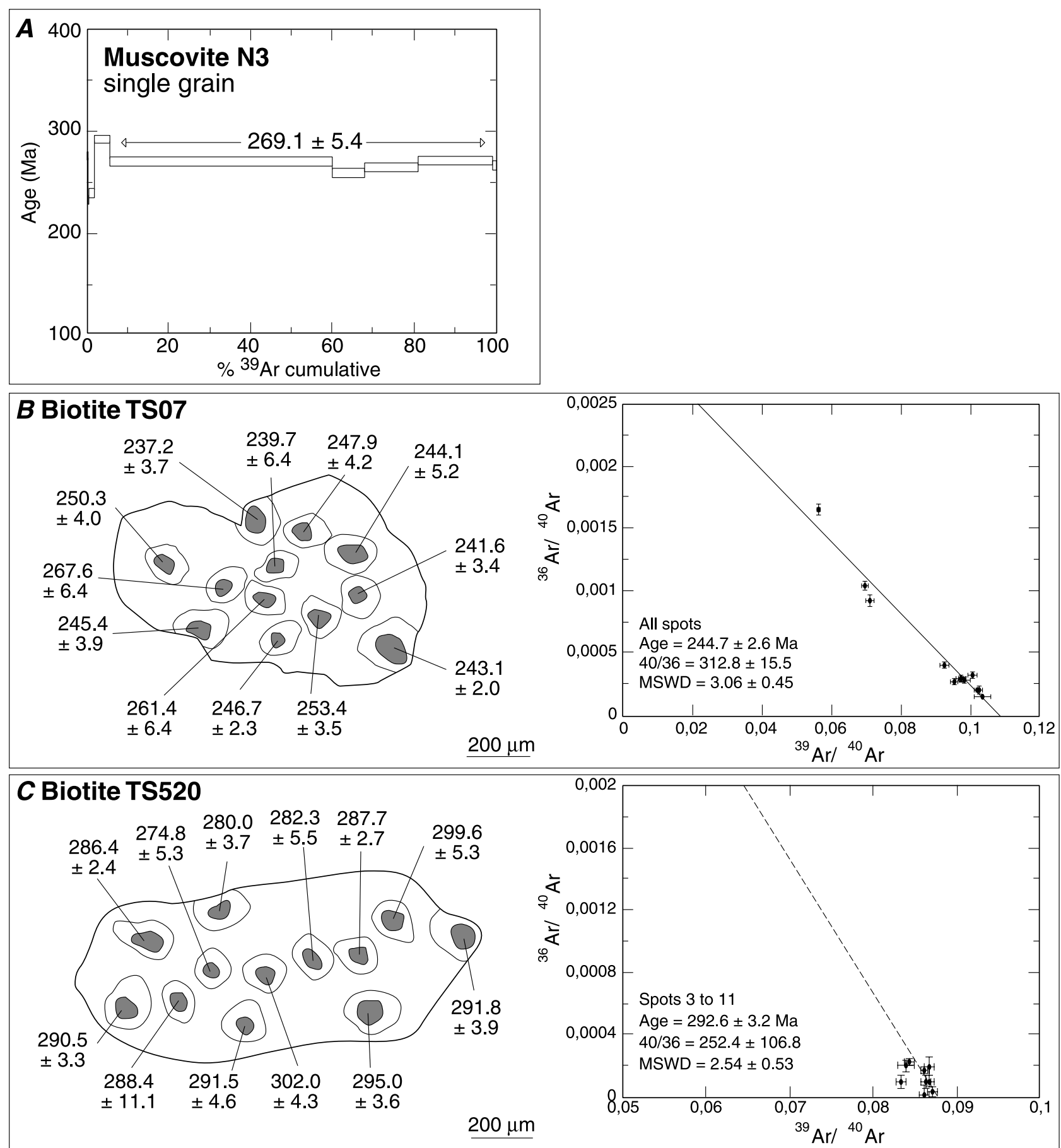

Figure 5. The ${ }^{40} \mathrm{Ar} /{ }^{39} \mathrm{Ar}$ dating results for samples N3, TS07, and TS520 for strike-slip motions in the central area of Tianshan range. Sample N3 comes from Shu et al. [1999a]. See Figure 3 for location.

droxylation, the age of $\sim 270$ Ma could be only a meaningless value intermediate between the Upper Permian and Carboniferous events [Hodges and Bowring, 1995]. However, there are still a lot of geochronological studies to do in the Tianshan range to understand clearly the chronology of the late Paleozoic strike-slip events.

\section{Ductile Strike-Slip Faults and Cooling History in the Chinese Altay}

[21] The northeastern border of the Junggar basin is mainly represented by the Altaid strike-slip system of central Asia of which the $2000 \mathrm{~km}$ long Irtysh-Erqishi 


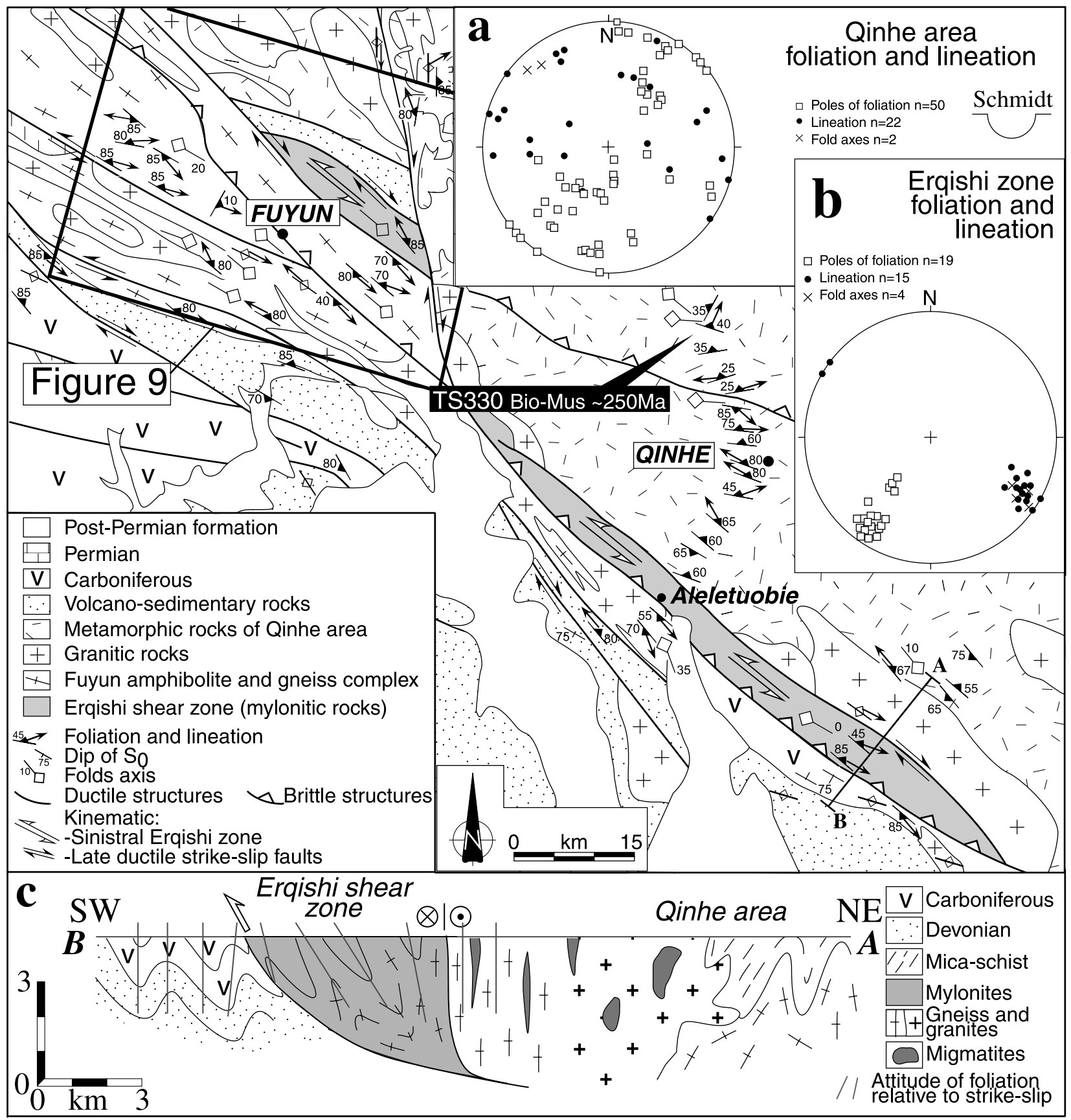

Figure 6. Structural sketch map of Erqishi sinistral shear zone and Qinhe area (modified from the geological map of Xinjiang Uygur Autonomous Region, scale 1:2,000,000 [Xinjiang Bureau of Geology and Mineral Resources, 1992]. Stereograms of foliations and lineations for (a) Qinhe and (b) Erqishi. (c) Simplified cross section of the Erqishi shear zone. Location of dated sample TS330 and of Figure 9 is indicated (modified from Laurent-Charvet et al. [2002]).

shear zone is an important member. In China it occurs between the south of Qinhe to the north of Altay city along a SE-NW trend. The shear zone separates a volcaniclastic and gneissic unit of supposed Proterozoic to Ordovician age to the northeast from the complex metamorphic zone of Fuyun to the southwest (Figure 6). The three areas studied below provide important data on the structure and the timing of the several strike-slip motion, which occurred in the Chinese Altay and on a larger scale in the eastern central Asia.

\subsection{Deformation in the Chinese Side of the Southwestern Margin of Siberia}

[22] In the northeastern area of the Chinese Altay Mountains a huge Paleozoic series of gneiss, metavolcanic, and 


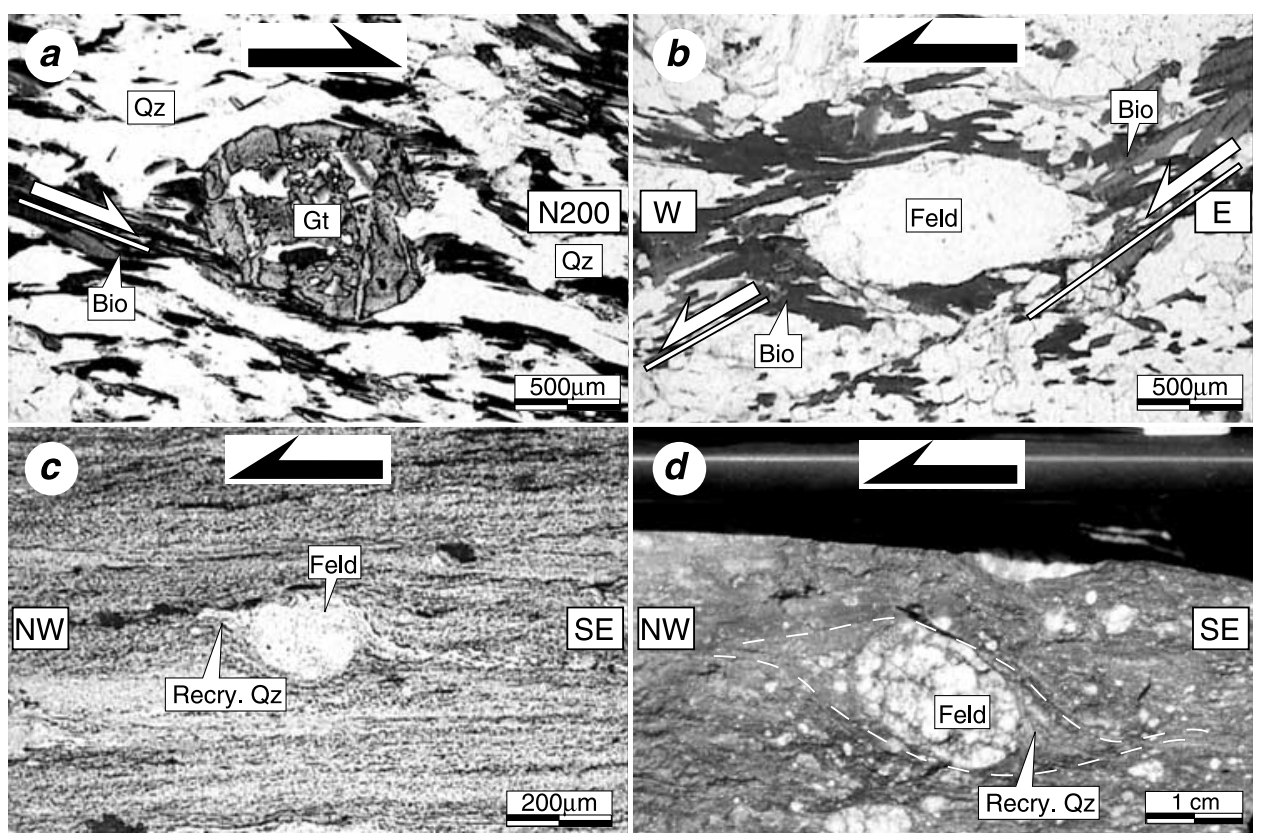

Figure 7. Examples of microstructures observed in the Qinhe area and in the Erqishi sinistral shear zone. (a) Biotite tails around garnet in an orthogneiss with a N20 dipping lineation, Qinhe area. Top-to-the-N200 shearing is indicated. (b) Shear bands underlined by biotites around a feldspar in an migmatitic gneiss with an horizontal lineation, Qinhe area. Top-to-the-W sense of shearing is indicated. (c) Sigmoid criteria made of a feldspar with recrystallized quartz tails in a metavolcanic mylonite, Erqishi shear zone. Sinistral sense of shearing is indicated. (d) Relict feldspar with asymmetric tails, Erqishi shear zone. Sinistral sense of shearing is indicated. For whole pictures, foliation is parallel to large side. Abbreviations are as follows: Bio, biotite; Feld, feldspar; Qz, quartz; Gt, garnet; and Recry. Qz, recrystallized quartz.

metasedimentary rocks is widely intruded by several plutons. Basement rocks consist of a lower unit of orthogneiss, paragneiss, and garnet-tourmaline-bearing migmatites and an upper unit of staurolite-bearing micaschists, schists, and a thick volcanosedimentary folded sequence. Metamorphic rocks are affected by a penetrative foliation that has an average N95 trend. Two main lineations are carried by the folded foliation (Figure 6a). First, a subhorizontal stretching lineation, associated with a top-to-the-west deformation is preserved in meter-scale shear zones. In these shear zones, criteria associated with the horizontal lineation are mainly recrystallized quartz sigmoids and feldspar with biotite tails (Figure 7b). These objects and associated minerals indicate a predominant top-to-the-west (or sinistral, depending of the foliation dip) deformation in high- to medium-temperature conditions. Second, an average N15 steeply dipping lineation related to a late thrusting event is well represented in the metamorphic formations. Several field shear criteria in migmatites, as overturned microfolds and quartz veins, point to a southward thrusting coeval with the north plunging lineation. In thin section, criteria are sigmoid objects, chloritized biotite shear bands, and mica tails around garnet and indicate a consistent top-to-the-south motion in mediumtemperature conditions (Figure 7a). This top-to-the-south shearing is associated with hectometer-scale folds. However, owing to the lack of radiometric and of field evidences in the Qinhe area the timing of the deformations remains debatable. In this study, one sample (TS330, shown on Figure 6) was dated by the ${ }^{40} \mathrm{Ar} /{ }^{39} \mathrm{Ar}$ method to compare the age of Qinhe area structuring with the timing of deformation in the nearby Fuyun and Erqishi shear zones (samples TS247, TS294, TS295, TS305, and TS310 shown on Figure 9).

[23] Sample TS330 is a micaschist with a N80-25N foliation and a lineation plunging to the N265. Biotite and muscovite form tails around feldspatic clasts and underline top-to-the-west shear bands. Kink bands occur in some muscovite crystals. Two methods of gas extraction were used on this sample. First, 14 spot fusion laser probe experiments were carried out on a single grain of nonchloritized biotite (Table 1). Apparent ages range between $231.1 \pm 2.5 \mathrm{Ma}$ and $275.9 \pm 3.4 \mathrm{Ma}$ with no systematic core versus rim distribution (Figure 8a). The atmospheric contamination is higher in the first five experiments $(17-43 \%)$ than in the subsequent analyses as in the case of the Tianshan samples. An isochron age of $261.4 \pm 3.2 \mathrm{Ma}$ has been obtained in the ${ }^{36} \mathrm{Ar} /{ }^{40} \mathrm{Ar}$ versus ${ }^{39} \mathrm{Ar} /{ }^{40} \mathrm{Ar}$ isotope correlation plot with an initial ${ }^{40} \mathrm{Ar} /{ }^{36} \mathrm{Ar}$ ratio of $271.1 \pm$ 26.1 (MSWD $=15.54 \pm 0.41$ ) (Figure 8a and Table 4). Second, a single grain muscovite was stepwise heated and gave a plateau age of $249.9 \pm 2.2 \mathrm{Ma}$ for $93 \%$ of the ${ }^{39} \mathrm{Ar}$ released and an integrated age of $249.8 \pm 2.2$ (Table 2 and Figure $8 \mathrm{~b}$ ). The atmospheric contamination is high in the three first steps $\left(80-11 \%\right.$ of ${ }^{40} \mathrm{Ar}$ atmospheric) and remains stable for the other ones. An intercept age of $248.8 \pm 2.2 \mathrm{Ma}$ has been obtained in the ${ }^{36} \mathrm{Ar} /{ }^{40} \mathrm{Ar}$ versus ${ }^{39} \mathrm{Ar} /{ }^{40} \mathrm{Ar}$ isotope correlation plot (Figure $8 \mathrm{~b}$ and Table 4) with a MSWD of 

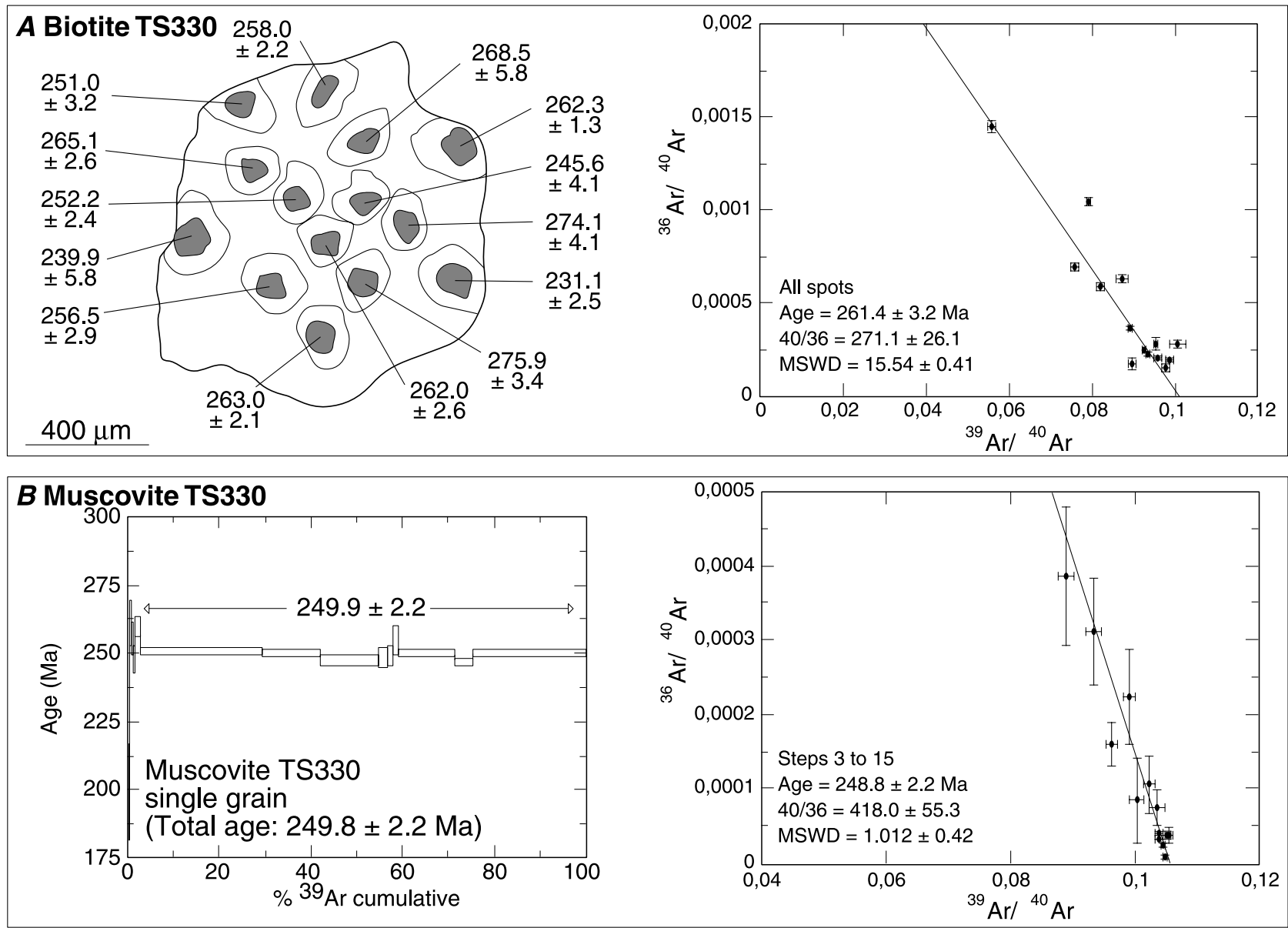

Figure 8. The ${ }^{40} \mathrm{Ar} /{ }^{39} \mathrm{Ar}$ dating results ${ }^{36} \mathrm{Ar} /{ }^{40} \mathrm{Ar}$ versus ${ }^{39} \mathrm{Ar} /{ }^{40} \mathrm{Ar}$ isotope correlation plots for sample TS330 in the Qinhe area. See Figure 6 for location.

$1.012 \pm 0.42$ and an initial ${ }^{40} \mathrm{Ar} /{ }^{36} \mathrm{Ar}$ ratio of $418.0 \pm 55.3$ that is likely to be indicative of an argon excess. In this sample, biotite may well have crystallized before $261 \mathrm{Ma}$ and was partially reopened during the muscovite crystallization at 250Ma. Another possibility is that the age of 261 Ma recorded by the biotite results from an argon excess in this mineral, so that the muscovite age of $250 \mathrm{Ma}$ is the only one that corresponds to the deformation age.

[24] Thus, in the area of Qinhe, two deformations were recognized by the structural studies. A southward deformation occurred in low temperature conditions, but there is no evidence concerning the age of this deformation. Owing to the important chloritization of the micas included in the gneiss and migmatite, which recorded this south verging shearing, no age could be measured. A top-to-the-west deformation also took place in the area $250 \mathrm{Myr}$ ago, coeval with the crystallization of muscovite and the likely remobilization of early biotites which may have formed during an earlier thermal event at $\sim 261 \mathrm{Ma}$ (Figure 8a and Table 4).

\subsection{Erqishi Shear Zone}

[25] The Erqishi sinistral NW-SE trending mylonitic shear zone (Figure 6) is of regional scale and stretches from the south of Qinhe to Kazakhstan (Figures 1 and 2) where it becomes the Irtysh shear zone [Melnikov et al., 1997; Mitrokhin et al., 1997]. It is 5 to $10 \mathrm{~km}$ wide and, according to Bulin et al. [1969], could reach a depth of $100 \mathrm{~km}$. This shear zone is presently bordered by late brittle dextral strikeslip faults and cut by southwestward brittle thrusts (Figures 6c and 9). Deformed rocks consist of mylonitic metatuffs, metasandstones, and andesitic schists of unknown age.

[26] In the mylonitic zone, rocks present a N120 trending foliation with a NE dip. The foliation carries a well-marked N120 trending horizontal stretching lineation (Figure 6b). On several outcrops, mylonites and ultramylonites exhibit southwestward tight overturned folds with 120SE15 axis parallel to lineation. These folds can be considered as Atype folds, characteristic of strong rate of deformation [Ramsay and Huber, 1987].

[27] The kinematic study of shear criteria associated with the stretching lineation points to a predominantly sinistral motion. Criteria are $\sigma$ - and $\delta$-type asymmetric objects (Figure 7c). Mylonites are also affected by sinistral shear bands. Deformed metasandstones contain "mica fish" structures, antithetical microfaulted feldspars in a quartzofeldspathic groundmass, and porphyroclasts with recrystallized quartz tails (Figure $7 d$ ). The $\sigma$ structures are typical of 


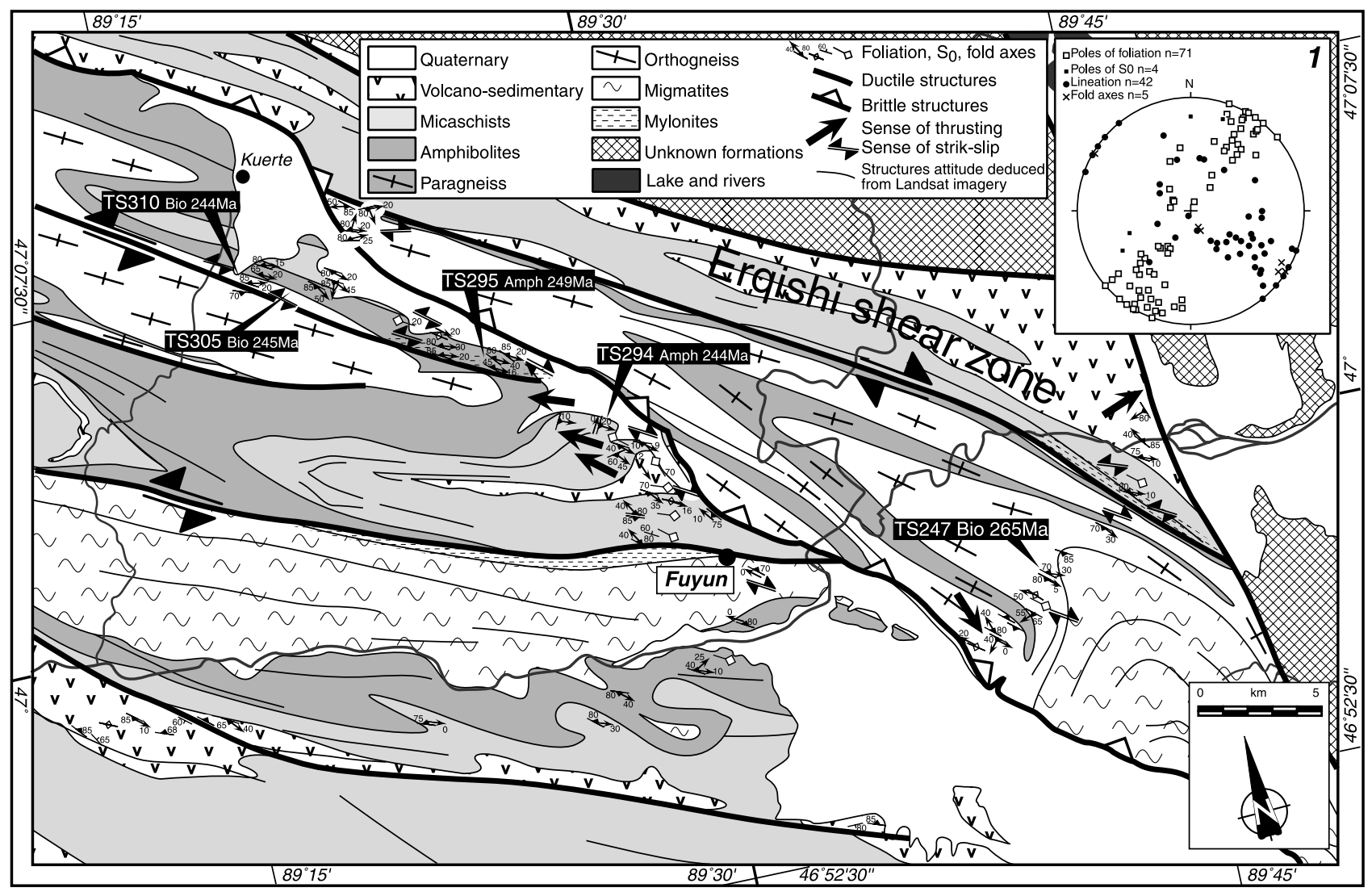

Figure 9. Detailed geological and structural map of the Fuyun area, drawn from 1:200,000 scale Landsat scenes and fieldwork observations. Inset labeled 1 is stereogram of foliations and lineations. Location of dated samples is shown.

low-strain shear zones where recrystallization rates are higher than rotation rates [Ramsay and Huber, 1987], while the $\delta$ structures are characteristic of higher shear strains where the rates of recrystallization are lower than that of rotation [Passchier and Simpson, 1986; Passchier and Trouw, 1996]. Thus, in the Erqishi mylonitic zone, the $\delta$ type structures can be considered as indicators of the maximum rate of shearing deformation.

[28] Some mylonite samples contain dextral criteria such as quartz porphyroclasts with recrystallized quartz tails. These dextral objects have not been reworked or deformed by sinistral ones and seem to be contemporaneous with the main sinistral motion [Laurent-Charvet et al., 2002]. They are interpreted as local traces of reverse shearing which commonly develops in mylonitic shearing associated with low-grade metamorphism [Hippertt and Tohver, 1999].

[29] Owing to the inaccurate petrology of the rocks cropping out along this shear zone in China, no ${ }^{40} \mathrm{Ar} /{ }^{39} \mathrm{Ar}$ dating has been performed to constrain the age of the sinistral motion. In Kazakhstan, Melnikov et al. [1998] obtained ages of about 280-290 Ma (Ar/Ar on single grain biotites). Duration of the transcurrent motion is still discussed, but the amplitude of displacement can have reached several hundred kilometers in regard of the depth and the length of this crustal structure. Sengör et al. [1993] pro- posed that the displacement reached $2000 \mathrm{~km}$ and suggested a Late Permian age for the main event.

\subsection{Complex Folded Shear Zone of Fuyun}

\subsubsection{Structural Study}

[30] After the studies of the Qinhe and Erqishi zones the structural study of the Fuyun area is important to understand the complex structuring of the Chinese Altay area and the chronology of the several deformations that structured this region. The complex metamorphic zone of Fuyun is a $15-$ $20 \mathrm{~km}$ wide folded zone located in the southwest of the Erqishi shear zone and extends from the southeast of Fuyun to Kazakhstan. The complex zone of Fuyun is cut in its center by a brittle southwestward thrust of unknown age (Figure 9). The southern limit of this metamorphic zone is marked by a strike-slip shear zone. Its northern border is a late dextral strike-slip ductile-to-brittle fault, which separates the Fuyun area from the Erqishi shear zone. Metamorphic formations consist of cordierite-garnet-bearing migmatites, orthogneisses, paragneisses, orthoamphibolites, micaschists, and greenschists facies metavolcanic and sedimentary rocks at the top of the series.

[31] Field and microscopic observations show that the deformation decreases from the bottom to the top of the 
metamorphic sequence. All the formations are affected by a regional foliation, which has an average direction of N120 and various high dips to NE and SW because of a horizontal N120 axis folding (Figure 9 inset labeled 1). Some outcrops, where foliation has a N10 direction and dips to the SE by $10^{\circ}-30^{\circ}$, are located in terminal bends of regional folds. In the deepest formations (gneisses and amphibolites) the foliation carries a stretching lineation (Ls) which remains constant in all the outcrops, whatever the foliation dipping is. This lineation roughly plunges to the N120 by $45^{\circ}-0^{\circ}$, depending if the foliation is subhorizontal or vertical (Figure 9 inset labeled 1).

[32] The upper formations, which outcrop in the synclinal bends, are affected by a weak deformation mainly represented by brittle-to-ductile structures. In the metavolcaniclastic rocks the only planar anisotropy that was observed is the folded stratigraphic layering. The axes of the numerous sheath and A-type folds that affect the formations constitute a lineation. These $L$ tectonics-type structures [Ramsay and Huber, 1987] correspond to disharmonic structures that accommodate the regional tight upright folding in the bends of folds. In the underneath micaschists the deformation progressively appears more significant, and lineation becomes a real stretching lineation that recorded the main shearing event. Owing to their petrology, schists do not contain interpretable microscopic sense indicators, but some outcrops display pluricentimeters-scale quartz veins and overturned folds that suggest a top-to-the-WNW motion.

[33] Beneath the micaschists, orthoamphibolites and gneisses present a more penetrative foliation. The $\sim$ N125 trending lineation is clearly a ductile stretching lineation $(L s)$. Protoliths of these orthoamphibolites were probably mafic or ultramafic rocks [ $L i$ and Bespaev, 1994]. The amphiboles were studied by electronic microprobe analysis, and results are synthesized in Figure 12c. Relicts of tschermakite may be inherited from an earlier thermal stage. Syntectonic Mg-hornblendes and actinolites, which crystallize in lower-temperature conditions than tschermakite [Dahl, 1996], underline shear indicators (such as shear bands) that formed during the northwest verging deformation. Thus, in a low SE dipping foliated amphibolite, which represents an anticlinal crest (Figure 10a), neohornblendes and actinolites underline shear bands and have crystallized in pressure shadow tails around relicts. All criteria indicate a top-to-the-WNW motion (Figure 10b). Amphibolites also occur as a north anticline limb with a NE high dipping foliation (sample TS295 on Figure 9). Lineation dips to the N125 of $20^{\circ}$, and associated criteria indicate a top-to-theWNW motion. This shearing is highlighted by sigmoid amphiboles, asymmetric pressure shadow tails around relicts, and shear bands underlined by hornblende and actinolite (Figure 10c). Few of these NE dipping amphibolites present chlorite crystallization in top-to-the-SE pressure shadow tails and ductile-to-brittle shear bands that reworked top-to-the-NW structures. This late low-temperature event is probably a dextral shearing that affected some zones after the main northwestward deformation.

[34] Orthogneisses were mainly observed in NE dipping anticline limbs in the south of Kuerte (Figure 9). Foliation has a N120 to N105 direction and dips to the NE by $75^{\circ}-$ $85^{\circ}$. Stretching lineation $(L s)$ is subhorizontal. Macroscopic structures indicate a top-to-the-NW motion such as centimeter-scale shear bands (Figure 10d). In thin sections all the criteria also attest for a top-to-the-NW motion. They are feldspars with mica tails, biotite shear bands and sigmoid quartz recrystallization around magmatic garnets (Figure 10e). Synkinematic biotite and muscovite and recrystallized quartz with subjoint and grain boundary migration features are characteristic of medium temperature deformation. In the eastern area of Fuyun some outcrops are formed of SW dipping orthogneiss affected by the N120 lineation. In these cases, shear bands and sigmoid objects indicate a sense of shearing which attests the top-to-the-NW motion (Figure 10f).

\subsubsection{The ${ }^{40} \mathrm{Ar} /{ }^{39} \mathrm{Ar}$ Dating}

[35] In an attempt to precise the chronology of successive deformation stages, five rocks from the Fuyun complex were selected for dating (Figure 9): two orthogneisses in the Fuyun metamorphic zone (TS305 and TS310), two mylonitic amphibolites (TS294 and TS295) from the core and the limb of a regional fold, and a migmatitic gneiss from the eastern end of the Fuyun zone (TS247).

[36] Sample TS247 is an orthogneiss made of feldspar, micas, and cordierite. It is characterized by a N130-80SW foliation carrying a 115SE5 lineation Ls. Associated kinematic criteria indicate a dextral shearing. They are mainly mica tails around feldspatic clasts. The textural ground is composed of quartz and biotite, which is parallel to the foliation and develops in pressure shadow tails. Few of these micas display traces of a limited chloritization. A single grain biotite was analyzed by 16 spot fusion laser probe experiments (Table 1). Ages scatter between $257.4 \pm$ 5.4 Ma and $281.4 \pm 8.0 \mathrm{Ma}$ (Figure 11a) with eight of them in a narrow range between $265.7 \pm 3.1 \mathrm{Ma}$ and $270.6 \pm 4.5$ Ma. The oldest ages are characterized by high ${ }^{40} \mathrm{Ar}$ atmospheric level (between 18\% and 60\%) and large error margins (about 6 to $9 \mathrm{Ma}$ ) compared to the other analyses with lower ${ }^{40} \mathrm{Ar}$ atmospheric contamination $(4-13 \%$ of ${ }^{40} \mathrm{Ar}$ ). In the ${ }^{36} \mathrm{Ar} /{ }^{40} \mathrm{Ar}$ versus ${ }^{39} \mathrm{Ar} /{ }^{40} \mathrm{Ar}$ isotope correlation plot an intercept age, which is the retained age (Table 4 and Figure 13e), of $265.6 \pm 2.5 \mathrm{Ma}$ is obtained with an initial ${ }^{40} \mathrm{Ar} /{ }^{36} \mathrm{Ar}$ ratio of $308.8 \pm 8.1$ and a MSWD of $2.2 \pm 0.38$ (Figure 11a).

[37] The cordierite-garnet-bearing orthogneiss TS305 has a N105-80N foliation with a 95E25 stretching lineation $L s$ and dextral microstructures as shear bands and micas tails. Only one generation of biotite, parallel to the foliation and present in tails around magmatic garnet and clasts, constitutes the textural ground with quartz and feldspar. No chloritization was observed. Thirteen spot fusion laser probe experiments were performed on a single biotite grain. Apparent ages range between $230.7 \pm 5.4$ and $260.1 \pm 9.6$ Ma (Table 1 and Figure 11b). Except for the two first spots, which contain $78 \%$ and $31 \%$ of surface related atmospheric ${ }^{40} \mathrm{Ar}$, they are characterized by a low atmospheric contamination (4-16\%). Eight spots bracketed between $242.4 \pm 7.2$ $\mathrm{Ma}$ and $247.4 \pm 5.6 \mathrm{Ma}$. In the ${ }^{36} \mathrm{Ar} /{ }^{40} \mathrm{Ar}$ versus ${ }^{39} \mathrm{Ar} /{ }^{40} \mathrm{Ar}$ isotope correlation plot an intercept age of $245.4 \pm 2.5 \mathrm{Ma}$ 


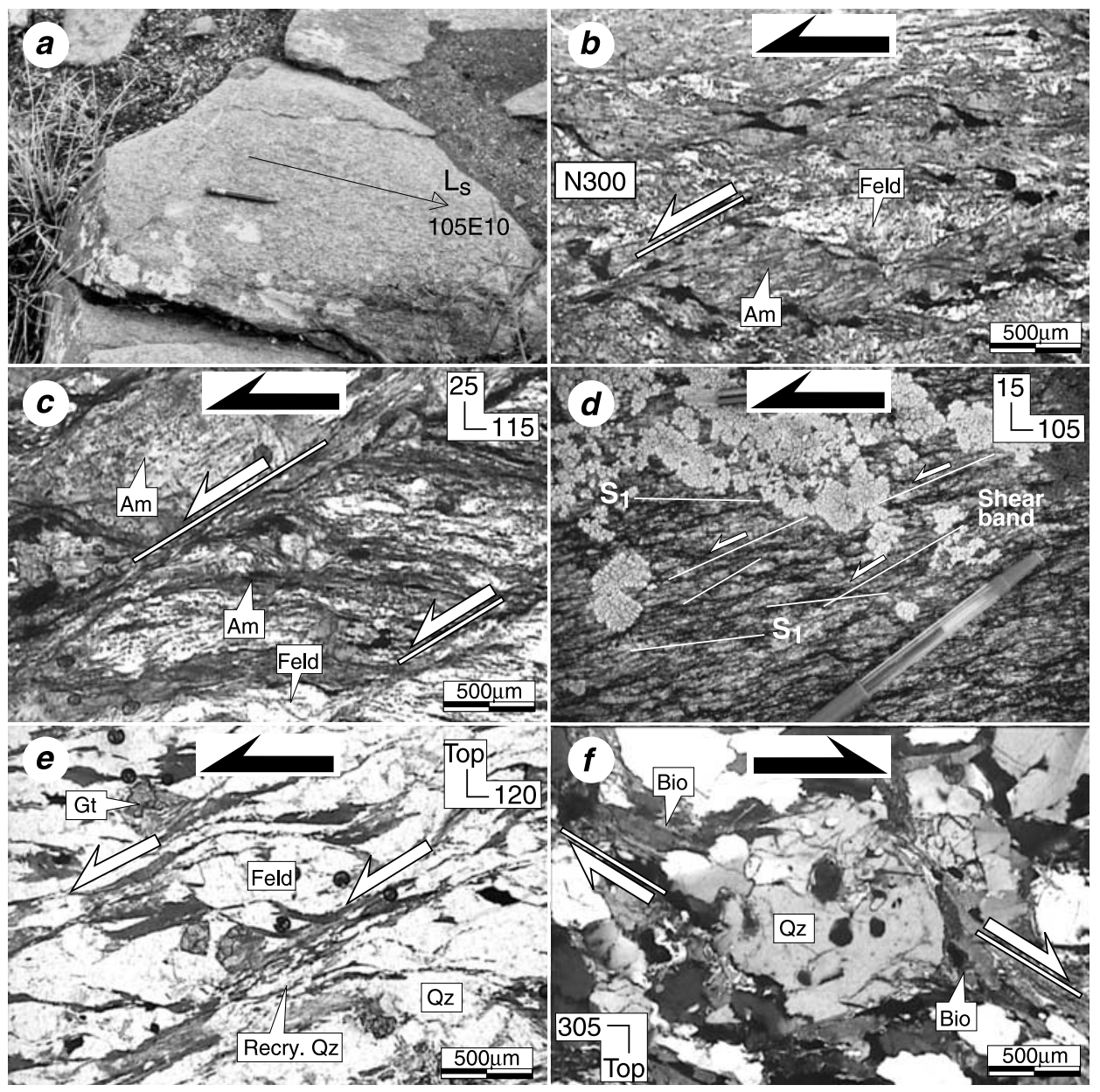

Figure 10. Microstructures in the Fuyun metamorphic zone. (a) Foliation dips to the NE Low dipping amphibolite with a 105E10 lineation. (b) Top-to-the-N300 shear bands in a subhorizontal foliated amphibolite. (c) Sinistral shear bands in a NE dipping amphibolite. (d) Centimeter-scale shear bands in a NW dipping gneiss. Sinistral sense of shearing is indicated. (e) Sinistral biotite shear bands in an orthogneiss. (f) Clast of quartz with biotite tails in a SW dipping foliated orthogneiss. Dextral sense of shearing is indicated. For Figures $10 \mathrm{a}-10 \mathrm{~d}$, foliation is parallel to large side. Abbreviations are as follows: Am, amphibole; Bio, biotite; Feld, feldspar; Qz, quartz; Gt, garnet; and Recry. Qz, recrystallized quartz.

(retained age, Table 4 and Figure 13b) is obtained with an initial ${ }^{40} \mathrm{Ar}{ }^{36} \mathrm{Ar}$ ratio of $292.3 \pm 7$ and a MSWD of $1.34 \pm$ 0.43 (Figure 11b).

[38] Sample TS310 is an orthogneiss made of quartz, feldspar, and few chloritized Fe-garnets (Alm. 70\%) with a N115-80NE foliation carrying a 105SE15 lineation Ls. Microstructures such as biotite tails around garnet indicate a top-to-the-N285 shearing. The textural ground is mainly characterized by quartz and biotite that suffered a starting chloritization. Two single biotite grains were extracted for spot fusion laser probe experiments. Seven spots on the first grain yield ages ranging from $142.4 \pm 27.8 \mathrm{Ma}$ to $228.0 \pm 10 \mathrm{Ma}$, with a very high ${ }^{40} \mathrm{Ar}$ contamination for all spots from $54 \%$ to $96 \%$ of the total ${ }^{40}$ Ar released (Table 1). This strong contamination is confirmed by the high position of the representative points of biotite 1 in the ${ }^{36} \mathrm{Ar} /{ }^{40} \mathrm{Ar}$ versus ${ }^{39} \mathrm{Ar} /{ }^{40} \mathrm{Ar}$ correlation plot (i.e., close to the Air intercept) (Figure 11c). The second biotite has eight ages scattering between $198.5 \pm 22.8 \mathrm{Ma}$ and $315.1 \pm 24.2 \mathrm{Ma}$ with a lower atmospheric contamination than for the first biotite $(8-43 \%)$. The ${ }^{36} \mathrm{Ar} /{ }^{40} \mathrm{Ar}$ versus ${ }^{39} \mathrm{Ar} /{ }^{40} \mathrm{Ar}$ isotope correlation plot compiles 14 spots (the one at $315 \mathrm{Ma}$ was removed) of the two biotites and gives an intercept age of $244.0 \pm 2.6 \mathrm{Ma}$ (Figure 11c and Table 4) for an initial ${ }^{40} \mathrm{Ar} /{ }^{36} \mathrm{Ar}$ ratio of $274.0 \pm 19$ and a MSWD of $3.74 \pm 0.63$. The age of $244 \mathrm{Ma}$ is interpreted as representing the cooling at about $300^{\circ}-350^{\circ} \mathrm{C}$ and coeval closure of the biotite.

[39] Mylonitic amphibolites TS294 and TS295 are made of amphiboles, albitized feldspar, minor clinozoisite (Czo $73-79 \%$ ) and chlorite. Two types of amphiboles were observed in these two samples. The first ones are high-Si Mg-hornblende to Fe-tschermakite that occur as relicts. The second ones surround the primary relicts and are mainly syntectonic low-Si Mg-hornblendes and actinolites (Figure 12c). In order to separately date the two gener- 

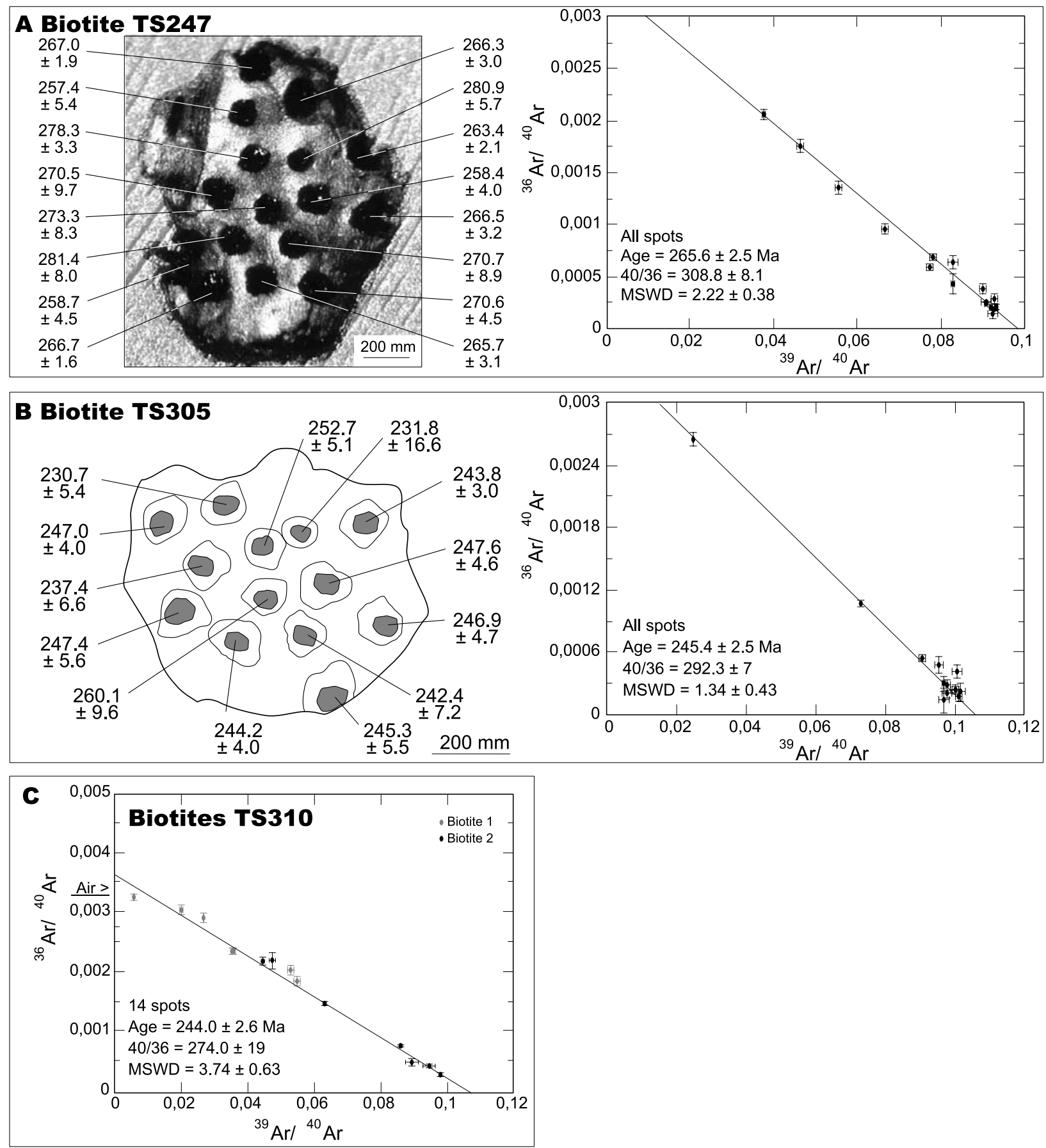

Figure 11. The ${ }^{40} \mathrm{Ar} /{ }^{39} \mathrm{Ar}$ dating results and corresponding ${ }^{36} \mathrm{Ar} /{ }^{40} \mathrm{Ar}$ versus ${ }^{39} \mathrm{Ar} /{ }^{40} \mathrm{Ar}$ isotope correlation plots for samples (a) TS247, (b) TS305, and (c) TS310 in the Fuyun metamorphic complex. See Figure 9 for location.

ations of amphiboles distinguished on chemical and textural ground, in situ laser probe fusion experiments were carried out on polished thin sections of about $1 \mathrm{~cm}^{2}$.

[40] Amphibolite TS294 has a N30-10E foliation carrying a N105SE10 stretching lineation $L s$. The textural ground is mylonitic, and microstructures indicate a top-
to-the-N285 motion for the deformation associated to the last thermal event (Figure 12d). Twelve spot fusions were carried out on secondary amphibole. Ages range between $195.8 \pm 6.8 \mathrm{Ma}$ and $279.7 \pm 12.8 \mathrm{Ma}$ (Table 3) with no systematic variation with the $\mathrm{Ca} / \mathrm{K}$ ratio deduced from the ${ }^{37} \mathrm{Ar} /{ }^{39} \mathrm{Ar}$ ratio. Among the spot fusions, six records give 

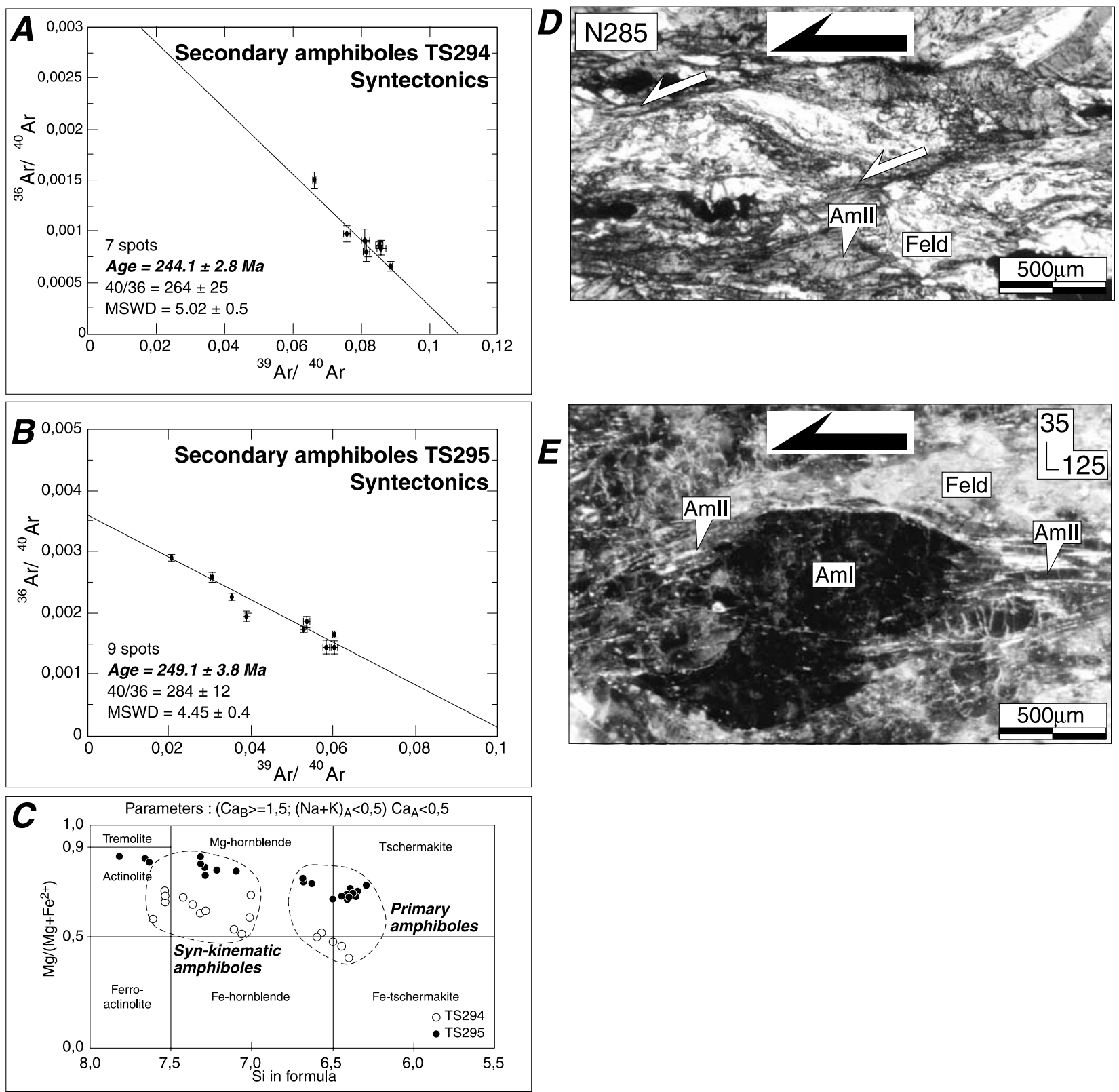

Figure 12. Amphibole ${ }^{40} \mathrm{Ar} /{ }^{39} \mathrm{Ar}$ dating in Fuyun metamorphic zone. (a) Sample TS294 ${ }^{36} \mathrm{Ar} /{ }^{40} \mathrm{Ar}$ versus ${ }^{39} \mathrm{Ar} /{ }^{40} \mathrm{Ar}$ isotope correlation plot. (b) Sample $\mathrm{TS} 295{ }^{36} \mathrm{Ar} /{ }^{40} \mathrm{Ar}$ versus ${ }^{39} \mathrm{Ar} /{ }^{40} \mathrm{Ar}$ isotope correlation plot. (c) Compositional diagram of the two types of amphibole in amphibolites TS294 and TS295 [Leake et al., 1997]. Microphotographs of shear criteria showing (d) a top-to-the-N285 deformation for sample TS294 and (e) a sinistral motion for sample TS295. Foliation is parallel to large side. See Figure 9 for location. Abbreviations are as follows: AmI, primary amphibole; AmII, secondary amphibole; and Feld, feldspar.

ages of 240-255 Ma. An intercept age of $244.1 \pm 2.8 \mathrm{Ma}$ has been obtained in the ${ }^{36} \mathrm{Ar} /{ }^{40} \mathrm{Ar}$ versus ${ }^{39} \mathrm{Ar} /{ }^{40} \mathrm{Ar}$ isotope correlation plot with an initial ${ }^{40} \mathrm{Ar} /{ }^{36} \mathrm{Ar}$ ratio of $264 \pm$ 25 and a MSWD of $5.02 \pm 0.5$ for the seven spots which have the lowest ${ }^{40} \mathrm{Ar}$ atmospheric yield (Figure 12a and Table 4).

[41] TS295 is a N120-85N foliated orthoamphibolite with some top-to-the-NW shear criteria along the 125SE20 lineation (Figure 12e). Fifteen spot fusion experiments were made on the section and give ages ranging between $140.4 \pm$ 36.1 Ma and $354.5 \pm 55.3 \mathrm{Ma}$, uncorrelated with the $\mathrm{Ca} / \mathrm{K}$ range (Table 3). As for sample TS294, owing to the low amount of potassium in amphibole, the ${ }^{40} \mathrm{Ar}$ atmospheric content is notably high and scatters between $85 \%$ and $40 \%$, resulting in important age errors. Seven spots with errors bars less than $15 \mathrm{Ma}$ are in the range 224-289 Ma. Together 
with two different spots with larger error bars, these spots yield an intercept age of $249.1 \pm 3.8$ Ma with an initial ratio of $284 \pm 12$ and a MSWD of $4.45 \pm 0.4$ (Figure 12b and Table 4). Younger ages are correlated with higher ${ }^{38} \mathrm{Ar} /{ }^{39} \mathrm{Ar}$ ratios, suggesting that argon was partially released from chlorine-rich domains during lasering. Ages older than 300 Ma probably result from an argon degassing from neighboring relict amphibole and/or from the presence of some excess argon in some minerals.

[42] However, despite the uncertainty concerning the nature and volume of material melted during these in situ laser probe experiments, and mainly the nature of the minerals behind those observed on the thin section surface, the present study of amphibolites in Fuyun yields intercept ages of $244.1 \pm 2.8 \mathrm{Ma}$ and $249.1 \pm 3.8 \mathrm{Ma}$ that are concordant with ages previously obtained on biotites $(245.4 \pm 2.5 \mathrm{Ma}$ and $244.0 \pm 2.6 \mathrm{Ma}$ for samples TS305 and TS310, respectively). On the basis of an ionic porosity model, Dahl [1996] calculated argon closure temperatures of $554^{\circ} \mathrm{C}$ and $483^{\circ} \mathrm{C}$ for the $\mathrm{Mg}$-hornblende and actinolite, respectively, while Harrison et al. [1985] suggested on the basis of diffusion experiments that biotite has a closure temperature between $280^{\circ} \mathrm{C}$ and $350^{\circ} \mathrm{C}$. The lack of significant age difference between biotites and amphiboles shows that the Fuyun metamorphic zone suffered probably a fast cooling from about $500^{\circ}-550^{\circ} \mathrm{C}$ to about $280^{\circ}-$ $350^{\circ} \mathrm{C}$ at about 250 and $245 \mathrm{Ma}$, respectively.

\subsubsection{Conclusion on Late Paleozoic Structures in the Fuyun Metamorphic Zone}

[43] Some dextral criteria were observed in a few south dipping formations, but the ${ }^{40} \mathrm{Ar} /{ }^{39} \mathrm{Ar}$ age of $265.6 \pm 2.5 \mathrm{Ma}$ obtained on sample TS247 is much too old to correspond exactly to the thermal event at $250 \mathrm{Ma}$ recognized in the Fuyun metamorphic zone (Figure 13e). Located on the northeastern border of the metamorphic zone, it is interpreted as a relict event of the sinistral deformation dated at 280-290 Ma in the Irtysh-Erqishi shear zone (Ar-Ar on micas by Melnikov et al. [1998]), which could migrate to the southwest through transcurrent motions between 265 and $245 \mathrm{Ma}$.

[44] The 250-245 Myr old event is well identified in the metamorphic formations where kinematic criteria related to this deformation are top-to-the-NW shear microstructures in all rocks whatever their foliation attitude. In north dipping limbs, criteria associated with stretching lineation point to the top-to-the-NW shearing in medium temperature conditions (Figures $13 \mathrm{a}-13 \mathrm{c}$ ). The ${ }^{40} \mathrm{Ar}{ }^{39} \mathrm{Ar}$ dating of this motion provides concordant ages of 249 to $245 \mathrm{Ma}$. Criteria associated with subhorizontal foliation in amphibolites also indicate a NW vergence of shearing (Figure 13d). This northwestward deformation has been dated at $244 \mathrm{Ma}$, which is concordant to that of shearing observed on NE dipping foliation. Thus the Fuyun zone underwent a sort of exhumation process, which was probably located between two main strike-slip shear zones. Squeezed between a north dextral fault and a south sinistral one, the area could have experienced a northwestward deformation $245 \mathrm{Myr}$ ago. Several reasons allow the assumption that this age $(\sim 245 \mathrm{Ma})$ is the strike-slip shearing final age.
When the deformations occur in low-temperature conditions $\left(300^{\circ}-400^{\circ} \mathrm{C}\right)$, it is possible to think that the obtained age from neoformed micas corresponds to their crystallization. When deformations occur in higher temperature range, micas begin to retain argon after the end of the strike-slip motion. Moreover, neoformed amphiboles, because of their higher closure temperatures, allow the dating of the end of the shearing when it occurs in hightemperature conditions. Thus the obtained ages certainly correspond to the end of the strike-slip activity. The exhumation process, which will be discussed in the conclusion, is associated with a fast cooling. It was probably due to the immediate thermal restabilization between sheared rocks and neighboring ones, but a local denudation could also participate to the fast cooling [Leloup et al., 2001a, 2001b].

[45] At the end of this shearing a compressive event induced the formation of tight upright folds, which affected all the metamorphic complex rocks. In the synclinal bends, disharmonic folds affect the upper metavolcaniclastic rocks in a weak ductile type deformation (Figures $13 \mathrm{f}$ and $13 \mathrm{~g}$ ). Finally, a dextral deformation occurred locally, in particular along the borders of the complex zone, in low-temperature conditions in the area. Chlorite tails and brittle shear bands underline the structures formed during this last stage. Its age is unknown, but the study of the satellite scenes shows that the Fuyun metamorphic zone borders have their directions secant to the foliation and to the regional folds ones, confirming the youngest age of this dextral strike-slip event.

\section{Discussion and Conclusion}

[46] In the Tianshan orogen the chronology of the different strike-slip deformation stages can be deciphered on the basis of several geological constraints. Field observations provide evidence confirming the late Paleozoic age of the strike-slip events: (1) The Carboniferous volcanic rocks of northern Tianshan and Late Carboniferous granites underwent the dextral ductile strike-slip deformation. (2) Upper Permian undeformed and unmetamorphosed rocks lie uncomformably on foliated Carboniferous rocks in the Hami area (eastern Tu-Ha basin).

[47] We propose that the strike-slip shearing may have reworked the main crustal discontinuities [e.g., Coleman, 1989; Ma et al., 1993; Shu et al., 1999a; Windley et al., 1990] at the end of the convergence between Junggar and central Tianshan in regional horizontal motions. The collisional thrust tectonics evolved into a transcurrent ductile dextral deformation, which probably accommodated the residual shortening by a first 290 Ma stage along the southern branch of the MTSZ in western Tianshan. A high-temperature conditions sinistral event occurred in the eastern Tianshan, but no chronological constraint support the assumption of a synchronous or earlier activity compared to the $290 \mathrm{Ma}$ event. Finally, a regional dextral strikeslip event occurred along the northern MTSZ and ended 245 Myr ago. Despite these results, there is still a lot of dating to do along the different branch of the MTSZ to confirm the 


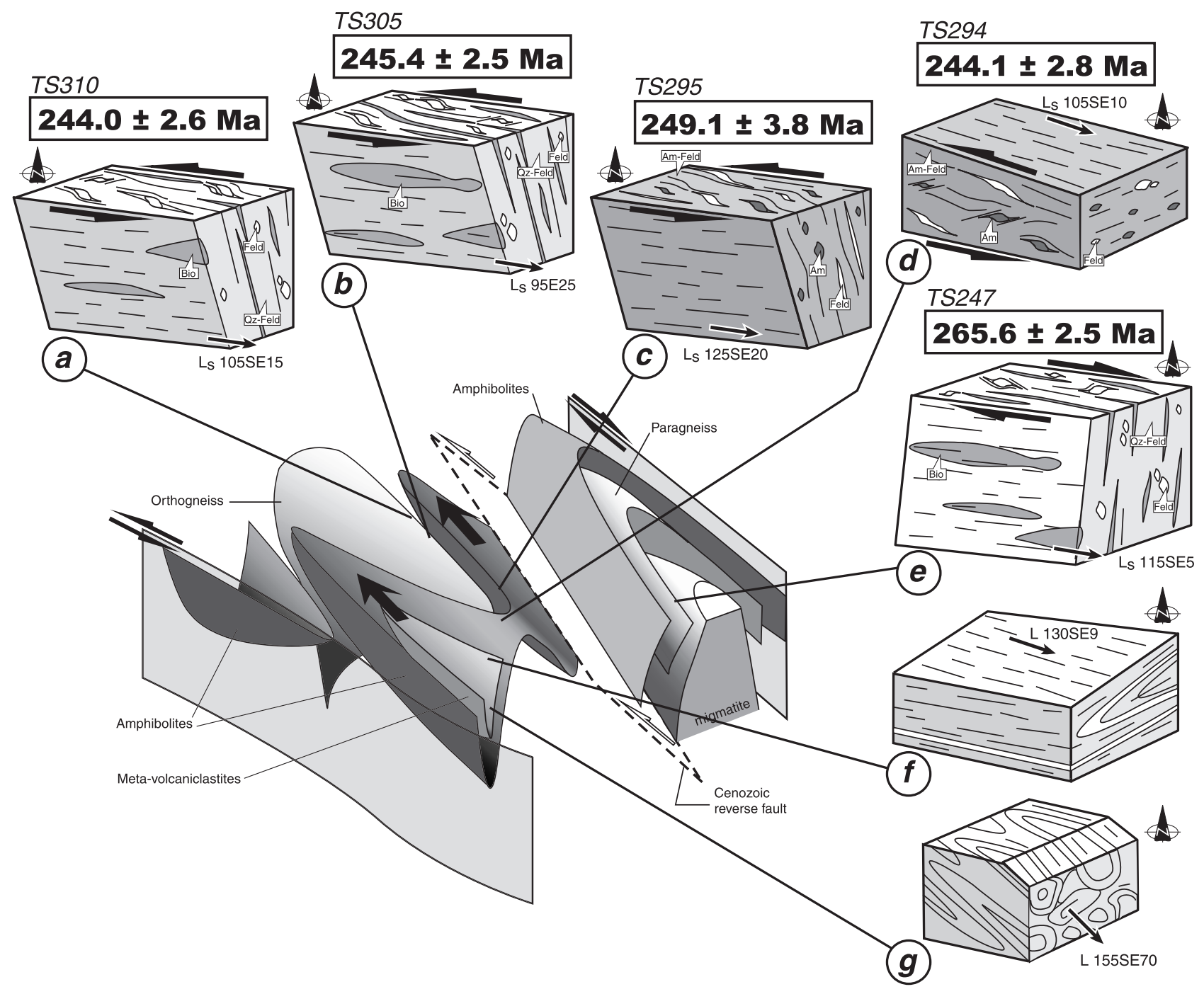

Figure 13. Three-dimensional block diagrams showing spatial and chronological relationships between folding, foliations, lineations, and shearing. Orthogneisses, samples (a) TS310 and (b) TS305, showing a top-to-the-NW sense of shearing on a NE dipping foliation. (c) Amphibolite (sample TS295) with a NE dipping foliation showing a top-to-the-NW deformation. (d) Amphibolite (sample TS294) with a SE low dipping foliation showing a top-to-the-N285 motion. (e) Gneiss (sample TS247) with a SW dipping foliation indicates a dextral shearing. (f) Metatuff with N110-10SW dipping foliation and a lineation plunging to the N130 of $9^{\circ}$. Isoclinal fold axes are parallel to lineation. (g) $L$ tectonics in a calcareous tuff. Presence of sheath folds parallels to the 159SE70 lineation. Abbreviations are as follows: Am, amphibole clast; Am-Feld, amphibolitofeldspatic groundmass; Bio, biotite ribbons or aggregates; Feld, feldspatic clast or recrystallized feldspar; and Qz-Feld, quartzofeldspatic groundmass.

assumption of the northward propagation of the strike-slip motion and the diachronism between the southern and the northern branches and to better constrain the different shearing stages between the eastern and the western Tianshan parts.

[48] In the Chinese Altay area several late Paleozoic ductile deformation stages took place between 290 and $245 \mathrm{Ma}$. The first deformation is exemplified by the Erqishi sinistral mylonitic zone. This crustal shearing started 290 Myr ago and built up all the southwestern boundary of the Altay-Sayan complex over more than $1000 \mathrm{~km}$. After this first event the transcurrent motion evolved into several structures such as top-to-the-W limited shear zones in the Qinhe area. Ages of 280-255 Ma were obtained in such a deformed gneiss. In this area a southward shearing was observed in gneiss and migmatites but no age was obtained. No evidence of this southward thrust motion was found in other studied areas. The Fuyun metamorphic zone displays a complex cooling and structural history. The structuring may have started before 265 Ma with a limited dextral shearing in the eastern part of the area. Then, the deformation probably migrated to the southwest 
Table A1. Location of Dated Samples and Summarize of Ages

\begin{tabular}{lllll}
\hline Samples & \multicolumn{1}{c}{ Area } & \multicolumn{1}{c}{ Lithology } & Latitude & Longitude \\
\hline N3 & North MTSZ (Mishigou) & muscovite bearing gneiss & N $42^{\circ} 24^{\prime}$ & E $88^{\circ} 29^{\prime}$ \\
TS07 & North MTSZ (Bindaban) & orthogneiss & - & - \\
TS520 & South MTSZ (Kumux) & orthogneiss & $\mathrm{N} 42^{\circ} 19^{\prime}$ & $\mathrm{E} 88^{\circ} 24^{\prime}$ \\
TS330 & Qinhe area & micaschist & $\mathrm{N} 46^{\circ} 47^{\prime} 40^{\prime \prime}$ & $\mathrm{E} 90^{\circ} 20^{\prime} 12^{\prime \prime}$ \\
TS247 & Fuyun metamorphic complex & orthogneiss & $\mathrm{N} 46^{\circ} 58^{\prime} 26^{\prime \prime}$ & $\mathrm{E} 89^{\circ} 39^{\prime} 56^{\prime \prime}$ \\
TS305 & Fuyun metamorphic complex & cordierite-garnet-bearing orthogneiss & $\mathrm{N} 47^{\circ} 09^{\prime} 11^{\prime \prime}$ & $\mathrm{E} 89^{\circ} 21^{\prime} 19^{\prime \prime}$ \\
TS310 & Fuyun metamorphic complex & garnet-bearing orthogneiss & $\mathrm{N} 47^{\circ} 07^{\prime} 18^{\prime \prime}$ & $\mathrm{E} 89^{\circ} 18^{\prime} 39^{\prime \prime}$ \\
TS294 & Fuyun metamorphic complex & mylonitic amphibolite & $\mathrm{N} 47^{\circ} 04^{\prime} 22^{\prime \prime}$ & $\mathrm{E} 89^{\circ} 28^{\prime} 05^{\prime \prime}$ \\
TS295 & Fuyun metamorphic complex & Mylonitic amphibolite & $\mathrm{N} 47^{\circ} 05^{\prime} 37^{\prime \prime}$ & $\mathrm{E} 89^{\circ} 26^{\prime} 08^{\prime \prime}$ \\
\hline
\end{tabular}

inducing a "squeezing" process 245 Myr ago. According to structural features and ${ }^{40} \mathrm{Ar} /{ }^{39} \mathrm{Ar}$ ages a northwestward deformation affected the metamorphic sequence before a regional tight folding in a relative global sinistral and compressive motion between Junggar and Siberian blocks. Indeed, the area of Fuyun is located on the southeastern margin of the Siberian block and could have recorded the important strains that probably occurred in the region between the two blocks. In the metamorphic sequence, temperature range and deformation increase from the top to the bottom but always indicate a top-to-the-N300 motion. Ages confirm the contemporaneity of sinistral, dextral, and northwestward displacements observed in the area, depending on whether the foliation dips to the northeast or southwest or is subhorizontal. This regional structuring seems to have induced a fast cooling, as the ${ }^{40} \mathrm{Ar} /{ }^{39} \mathrm{Ar}$ dating display. As it was shown in the Ailao Shan-Red River shear zone [Leloup et al., 1995, 2001a, 2001b], such a cooling rate resulted from the strike-slip motions, which induced the exhumation of rocks. Moreover, the cooling may be fast in the studied shear zones because of their limited width, which induced a fast thermal restabilization with the nonsheared surrounding rocks. Another process that probably participated in the fast cooling could be local denudations in a compressive environment like in the Ailao Shan-Red River shear zone [Leloup et al., 2001a]. Thus the structural and geochronological study of the Fuyun area, and also of the Tianshan, could be complete in the future by precise thermobarometric estimations on each shear zone.

[49] The structural and ${ }^{40} \mathrm{Ar} /{ }^{39} \mathrm{Ar}$ studies on transcurrent structures in eastern Xinjiang show that the late Paleozoic strike-slip faults can be interpreted as an intracontinental adjustment due to relative motions between all the different blocks making eastern central Asia after the Carboniferous thrusting stage. We make the assumption that, at the end of Carboniferous, the Tarim block continued to move forward to the north. The Tarim motion evolved into a northwestward direction, allowing the accommodation of strains along the southern MTSZ then the northern MTSZ and by strike-slip faults in the Chinese Altay. The tectonic development of north Xinjiang illustrates a transition between two styles of convergence and shortening accommodation. The deformation that occurred in the area during middle Paleozoic evolved into a Permian horizontal ductile deformation accommodating the residual shortening along the continen- tal scale structures (e.g., the Erqishi-Irtysh shear zone and the MTSZ) in eastern central Asia.

\section{Appendix A: The ${ }^{40} \mathrm{Ar} /{ }^{39}$ Ar Dating Analytical Procedure}

[50] During this study, owing to large grain size of micas in most samples (see descriptions of the samples in Table A1), we preferentially realized spot fusion on biotite and amphibole single grains using a laser probe operating in the semipulsed mode [Monié et al., 1997]. Only two single grains of muscovite were heated in a stepwise fashion using a continuous mode of laser emission. The samples were irradiated in the McMaster nuclear reactor (Canada) together with different flux monitors including $\mathrm{MMHb}-1$ $(520.4 \pm 1.7 \mathrm{Ma})$ and HD-B1 $(24.21 \pm 0.32 \mathrm{Ma})$. For this reactor the following correction factors for argon nuclear interferences were applied: $\left({ }^{36} \mathrm{Ar} /{ }^{37} \mathrm{Ar}\right)_{\mathrm{Ca}}=0.000254$, $\left({ }^{39} \mathrm{Ar} /{ }^{37} \mathrm{Ar}\right)_{\mathrm{Ca}}=0.000651$, and $\left({ }^{40} \mathrm{Ar} /{ }^{39} \mathrm{Ar}\right)_{\mathrm{K}}=0.0156$. Results are synthesized in Table 1 for spot fusion laser probe experiments, Table 2 for step-heating analysis, and Table 3 for spot fusion laser probe experiments on amphibolite polished sections. The analytical device is comparable to that described by Dalrymple [1989] and consists of (1) a multiline continuous $6 \mathrm{~W}$ argon-ion laser with two main wavelengths of 488 and $514 \mathrm{~nm}$; (2) a beam shutter for selection of exposure times, typically $30 \mathrm{~ms}$ for spot fusions; (3) an optical device to focus the laser beam down to a minimum impact diameter of $20 \mu \mathrm{m}$; (4) a small inlet line for the extraction and purification of gases; and (5) a MAP 215-50 noble gas mass spectrometer equipped with a Nier source and a Johnston MM1 electron multiplier. The gain between the Faraday cup and multiplier at $2 \mathrm{kV}$ is close to 200. Each analysis involves $5 \mathrm{~min}$ for gas extraction and cleaning and $15 \mathrm{~min}$ for data acquisition. System blanks were evaluated every three experiments and ranged from 3 $\times 10^{-12} \mathrm{cc}$ for ${ }^{40} \mathrm{Ar}$ to $6 \times 10^{-14} \mathrm{cc}$ for ${ }^{36} \mathrm{Ar}$. Isotopic corrections, age, and error calculations were made according to McDougall and Harrison [1988]. Mass discrimination was calculated on the basis of a ${ }^{40} \mathrm{Ar} /{ }^{36} \mathrm{Ar}$ ratio of $283.69 \pm 3.62$ measured on an atmospheric argon aliquot. The ages of individual steps or spots were calculated assuming an initial ${ }^{40} \mathrm{Ar} /{ }^{36} \mathrm{Ar}$ ratio of 295.5.

[51] Acknowledgments. The authors are grateful to the supports of the China 973 Project "Central Asian-type Orogeny and Metalliza- 
tion in the western China" (grants $\mathrm{n}^{\circ}$ 2001CB409804), the Natural Science Foundation of China (grants 49772151 and 49832040) and the Department of Earth Sciences of Nanjing University (China) which provided logistic support for field trips. B. Natal'in and the other reviewer also have our thanks for their important and constructive comments.

\section{References}

Allen, M. B., B. F. Windley, and C. Zhang, Paleozoic collisional tectonics and magmatism of the Chinese Tienshan, central Asia, Tectonophysics, 220, $89-$ 115,1992 .

Allen, M. B., B. F. Windley, C. Zhang, and J. H. Guo, Evolution of the Turfan basin, Chinese central Asia, Tectonics, 12, 889-896, 1993.

Allen, M. B., A. M. C. Sengör, and B. A. Natal'in, Junggar, Turfan and Alakol basins as Late Permian to? Early Triassic extensional structures in a sinistral shear zone in the Altaid orogenic collage, central Asia, J. Geol. Soc. London, 152, 327-338, 1995.

Allen, M. B., S. J. Vincent, and P. J. Wheeler, Late Cenozoic tectonics of the Kepingtage thrust zone: Interactions of the Tien Shan and Tarim basin, northwest China, Tectonics, 18, 639-654, 1999.

Avouac, J. P., and P. Tapponnier, Kinematic model of active deformation in central Asia, Geophys. Res. Lett., 20, 895-898, 1993.

Avouac, J. P., P. Tapponnier, M. Bai, H. You, and G. Wang, Active thrusting and folding along the northern Tien Shan and late Cenozoic rotation of the Tarim relative to Dzungaria and Kazakhstan, J. Geophys. Res., 98, 6755-6804, 1993.

Berzin, N., R. G. Coleman, N. L. Dobretsov, L. P. Zonenshain, X. C. Xiao, and E. Z. Chang, Geodynamic map of the western part of the Paleoasian ocean, Russ. Geol. Geophys., 35, 5-22, 1994.

Bulin, N. K., E. A. Pronyaeva, and V. I. Bubnova, Deep structure of the SW-Altay according to seismic data, Sov. Geol., 4, 97-109, 1969.

Burchfiel, B. C., E. T. Brown, Q. D. Deng, X. Y. Feng, J. Li, P. Molnar, J. B. Shi, Z. M. Wu, and H. C. You, Crustal shortening on the margins of the Tien Shan, Xinjiang, China, Int. Geol. Rev., 41, 665-700, 1999.

Burtman, V. S., Structural geology of the Variscan Tien Shan, USSR, Am. J. Sci. 275A, 157-186, 1975.

Charvet, J., S. Laurent-Charvet, and L. S. Shu, Continental accretion in central Asia: Paleozoic geotectonic evolution of Tianshan belt, Xinjiang, NW China (abstract), in 31st International Geological Congress [CD-ROM], vol. 1, edited by H. Chaves, Int. Union of Geol. Sci., Ottawa, Ont., Canada, 2000.

Che, Z. C., H. F. Liu, and L. Liu, The Formation and Evolution of the Central Tianshan Orogenic Belt, 135 pp., Geol. Publ. House, Beijing, 1994.

Chen, C. M., H. F. Lu, D. Jia, D. S. Cai, and S. M. Wu, Closing history of the southern Tianshan oceanic basin, western China: An oblique collisional orogeny, Tectonophysics, 302, 23-40, 1999.

Chen, Z. C., Forming and Evolution of the Middle Tianshan Orogenic Belt, Geol. Publ. House, Beijing, 1994.

Coleman, R. G., Continental growth of northwest China, Tectonics, 8, 621-635, 1989.

Cui, K., Research on the structural deformation and ductile shear zones of the western Tianshan, Ph.D. thesis, 126 pp., Nanjing Univ., Nanjing, China, 1995.

Cunningham, W. D., B. F. Windley, D. Dorjnamjaa, J. Badamgarov, and M. Saandar, A structural transect across the Mongolian Western Altai: Active transpressional mountain building in central Asia, Tectonics, 15, 142-156, 1996.

Dahl, P. S., The effects of composition on retentivity of argon and oxygen in hornblende and related amphiboles: A field-tested empirical model, Geochim. Cosmochim. Acta, 60, 3687-3700, 1996.
Dalrymple, G. B., The GLM continuous laser system for 40Ar/39Ar dating: Description and performance characteristics, in New Frontiers in Stable Isotopic Research: Laser Probes, Ion Probes, and SmallSample Analysis, edited by W. C. Shanks, and R. E. Criss, U.S. Geol. Surv. Bull., 3, 89-96, 1989. Dehandschutter, B., D. Delvaux, and A. Boven, The Lake Teletsk tectonic depression (Altai): New kinematic data and chronological relations, in Rapport Annuel 1995 and 1996, report, pp. 147-167, Dép. de Géol. Min., Mus. R. Afr. Cent., Tervuren, Belgium, 1997.

Dobretsov, N. L., N. Berzin, and M. Buslov, Opening and tectonic evolution of the Paleo-Asian Ocean, Int. Geol. Rev., 37, 335-360, 1995.

Gao, J., and R. Klemd, Eclogite Occurrences in the southern Tianshan high-pressure belt, Xinjiang, western China, Gondwana Res., 3, 33-38, 2000.

Gao, J., G. Q. He, M. S. Li, X. C. Xiao, Y. Q. Tang, J. Wang, and M. Zhao, The mineralogy, petrology, metamorphic PTDt trajectory and exhumation mechanism of blueschists, south Tianshan, northwestern China, Tectonophysics, 250, 151-168, 1995.

Gao, J., M. S. Li, X. C. Xiao, Y. Q. Tang, and G. Q. He, Paleozoic tectonic evolution of the Tianshan Orogen, northwestern China, Tectonophysics, 287, 213-231, 1998.

Gao, J., L. F. Zhang, and S. W. Liu, The 40Ar/39Ar age record of formation and uplift of the blueschists and eclogites in the western Tianshan Mountains, Chin. Sci. Bull., 45(11), 1047-1051, 2000.

Guo, Z. J., R. S. Ma, L. Z. Guo, and Y. S. Shi, A comparative study on three ophiolitic melange belts in eastern Xinjiang, Geol. Rev., 39, 236-247, 1993.

Han, B. F., S. G. Wang, B. M. Jahn, D. W. Hong, H. Kagami, and Y. L. Sun, Depleted-mantle source for the Ulungur River A-type granites from north Xinjiang, China: Geochemistry and Nd-Sr isotopic evidence, and implications for Phanerozoic crustal growth, Chem. Geol., 138, 135-159, 1997.

Han, B. F., G. Q. He, and S. G. Wang, Postcollisional mantle-derived magmatism and vertical growth of the continental crust in north Xinjiang, Geol. Rev., 44, 396-406, 1998

Harrison, T. M., I. Duncan, and H. Mc Dougall, Diffusion of ${ }^{40} \mathrm{Ar}$ in biotite: Temperature, pressure and compositional effects, Geochim. Cosmochim. Acta, 49, 2461-2468, 1985

Hendrix, M. S., T. A. Dumitru, and S. A. Graham, Late Oligocene-early Miocene unroofing in the Chinese Tian Shan: An early effect of the India-Asia collision, Geology, 22, 487-490, 1994.

Hippertt, J., and E. Tohver, On the development of zones of reverse shearing in mylonitic rocks, J. Struct. Geol., 21, 1603-1614, 1999.

Hodges, K. V., and S. A. Bowring, ${ }^{40} \mathrm{Ar} /{ }^{39} \mathrm{Ar}$ thermochronology of isotopically zoned micas: Insights from the southwestern USA Proterozoic orogen, Geochim. Cosmochim. Acta, 59, 3205-3220, 1995.

Hu, A., G. Zhang, and Q. Zhang, The basement age of Tianshan belt and the $\mathrm{Nd}$ isotopic constraining on crustal accretion, Sci. China, 20, 104-112, 1999.

Kwon, S. T., G. R. Tilton, R. G. Coleman, and Y. Feng, Isotopic studies bearing on the tectonics of the wes Junggar region, Xinjiang, China, Tectonics, 8 , 719-727, 1989 .

Laurent-Charvet, S., Accrétions continentales en Asie centro-orientale: Evolution géodynamique et structurale du Tianshan et du Junggar oriental (nord-ouest Chine) au Paléozoïque, Ph.D. thesis, 384 pp. Univ. d'Orléans, Orléans, France, 2001.
Laurent-Charvet, S., J. Charvet, and L. S. Shu, Late Palaeozoic strike-slip faults around Junggar basin, Xinjiang, NW China, in 31st International Geological Congress [CD-ROM], vol. 1, edited by H. Chaves, Int. Union of Geol. Sci., Ottawa, Ont. Canada, 2000a.

Laurent-Charvet, S., J. Charvet, L. S. Shu, R. S. Ma and H. F. Lu, Accrétion continentale en Asie centro-orientale: Evolution géotectonique du Xinjiang oriental au Paléozoïque, NW Chine, paper presented at 18ème Réunion des Sciences de la Terre, Soc. Geol. de France, Paris, 2000b.

Laurent-Charvet, S., P. Monié, J. Charvet, and L. S. Shu, New kinematic and Ar-Ar data on strike-slip ductile shear zones around Junggar basin, Xinjiang, NW China (abstract), in Journal of Conference EUG XI, [CD-ROM], vol. 6, Cambridge, Strasbourg, France, 2001.

Laurent-Charvet, S., J. Charvet, L. S. Shu, R. S. Ma, and H. F. Lu, Paleozoic late collisional strike-slip deformations in Tianshan and Altay, eastern Xinjiang, NW China, Terra Nova, 14(4), 249-256, 2002.

Leake, B. E., et al., Nomenclature of amphiboles; Report of the Subcommittee on Amphiboles of the International Mineralogical Association Commission on New Minerals and Mineral Names, Can. Mineral., 9, 623-651, 1997.

Leloup, P. H., R. Lacassin, P. Tapponnier, U. Schärer, D. Zhong, X. Liu, L. S. Zhang, S. C. Ji, and T. T. Phan Trong, The Ailao Shan-Red River shear zone (Yunnan, China), Tertiary transform boundary of Indochina, Tectonophysics, 251, 3-84, 1995.

Leloup, P. H., N. Arnaud, R. Lacassin, J. R. Kienast, T. M. Harrison, T. T. Phan Trong, A. Replumaz, and P. Tapponnier, New constraints on the structure, thermochronology and timing of the Ailao Shan-Red River shear zone, SE Asia, J. Geophys. Res., 106, 6683-6732, 2001a.

Leloup, P. H., R. Lacassin, P. Tapponnier, and T. M. Harrison, Comment on "Onset timing of left-lateral movement along the Ailao Shan-Red River shear zone: ${ }^{40} \mathrm{Ar} /{ }^{39} \mathrm{Ar}$ dating constraint from the Nam Dinh area, northeastern Vietnam" by Wang et al., 2000, J. Asian Earth Sciences, 18, 281-292, J. Asian Earth Sci., 20(1), 95-99, 2001 b.

Li, T. D., and H. A. Bespaev, Comparative study of geology and mineralization of Altai multimetal ore belt between China and Kazakhstan, in The Office of the National Key Project "305" in Uygur Autonomous Region of Xinjiang and Institute of Geology, edited by K. A. O. Sciences, pp. 7-17, Kazakhstan Acad. of Sci., Alma Ata, 1994.

Liu, W., Whole rock isochron ages of plutons crustal movements and evolution of tectonic setting in the Altay Mts. Xinjiang, Geosci. Xinjiang, 2, 35-50, 1993

Liu, X., D. Fu, J. X. Yao, X. Z. Ding, Y. Wang, S. A Graham, E. Z. Chang, E. R. Sobel, S. Z. Wu, and Y. Yan, Tectonic evolution of Tarim plate and surrounding areas since late Paleozoic, Cont. Dyn., 1, $110-122,1996$

Ma, R. S., S. F. Ye, C. Y. Wang, and G. B. Liu, Framework and evolution in the east Tianshan orogenic belt, Geosci. Xinjiang, 1, 21-36, 1990.

Ma, R. S., C. Y. Wang, and S. F. Ye, Tectonic Framework and Crustal Evolution of Eastern Tianshan Mountains, 225 pp., Publ. House of Nanjing Univ., Nanjing, China, 1993.

Ma, R. S., L. S. Shu, and J. Q. Sun, Tectonic Evolution and Metallogeny of Eastern Tianshan Mountains, 202 pp., Geol. Publ., Beijing, 1997. 
McDougall, I., and T. M. Harrison, Geochronology and Thermochronology by the ${ }^{40} \mathrm{Ar}{ }^{39} \mathrm{Ar}$ Method, 212 pp., Oxford Univ. Press, New York, 1988.

Melnikov, A., et al., Late Paleozoic-early Mesozoic sinistral movement along the Irtysh shear zone, NEKazakhstan, paper presented at Tectonic Studies Group Annual General Meeting, Univ. of Durham, Durham, U.K., 1997.

Melnikov, A., A. Travin, A. Plotnikov, L. Smirnova, and $\mathrm{K}$. Theunissen, Kinematics and $\mathrm{Ar} / \mathrm{Ar}$ geochronology of the Irtysh Shear zone in the NE Kazakhstan, in $I G C P 420$, edited by B. M. Jahn and D. W. Hong, 60 pp., Int. Union of Geol. Sci., Ottawa, Ont., Canada, 1998.

Mitrokhin, D., A. Kazansky, K. Theunissen, and N. Berzin, Paleomagnetic and kinematic characteristics of the Irtysh shear zone near Predgornoye (East Kazakhstan): Preliminary results, in Rapport Annuel 1995 and 1996, pp. 187-201, Dép. de Géol. Min., Mus. R. Afr. Cent., Tervuren, Belgium, 1997.

Molnar, P., and P. Tapponnier, Cenozoic tectonics of Asia: Effects of a continental collision, Science, 189, 419-426, 1975.

Monié, P., R. Caby, and M. H. Arthaud, The Neoproterozoic Brasiliano orogeny in northeast Brazil: ${ }^{40} \mathrm{Ar} /{ }^{39} \mathrm{Ar}$ and petrostructural data from Ceará, Precambrian Res., 81, 241-264, 1997.

Natal'in, B. A., and A. M. C. Sengör, The tectonic setting of the Tien Shan within the Altaid orogenic belt, Geol. Soc. Am. Abstr. Programs, A464, 1994.

Nelson, M. R., R. McCaffrey, and P. Molnar, Source parameters for eleven earthquakes in the Tien Shan, central Asia: Determined by $P$ and $\mathrm{SH}$ waveform inversion, J. Geophys. Res., 92, 12,629-12,648, 1987.

Nishidai, T., and J. L. Berry, Geological interpretation and hydrocarbon potential of the Turpan basin (NW China) from satellite imagery, paper presented at Eighth Thematic Conference on Geologic Remote Sensing, Denver, Colo., 1991.
Passchier, C. W., and C. Simpson, Porphyroclast systems as kinematic indicators, J. Struct. Geol., 8 , $831-843,1986$.

Passchier, C. W., and R. A. J. Trouw, Microtectonics, 289 pp., Springer-Verlag, New York, 1996.

Ramsay, J. G., and M. I. Huber, The Techniques of Modern Structural Geology, vol. 2, Folds and Fractures, 250 pp., Academic, San Diego, Calif., 1987.

Ritz, J.-F., E. T. Brown, D. L. Bourlès, H. Philip, A. Schlupp, G. M. Raisbeck, F. Yiou, and B. Enkhtuvshin, Slip rates along active faults estimated with cosmic-ray-exposure dates: Application to the Bogd fault, Gobi-Altaï, Mongolia, Geology, 23 1019-1022, 1995.

Sengör, A. M. C., and B. A. Natal'in, Paleotectonics of Asia: Fragments of a synthesis, in The Tectonic Evolution of Asia, edited by Y. An and T. M. Harrison, pp. 486-640, Cambridge Univ. Press, New York, 1996.

Sengör, A. M. C., B. A. Natal'in, and V. S. Burtman, Evolution of the Altaid tectonic collage and Paleozoic crustal growth in Eurasia, Nature, 364, 299 307, 1993.

Shu, L. S., C. Y. Wang, and R. S. Ma, Granulite relics and pyroxene-facies ductile deformation in the northern boundary of the Southern Tianshan, Sci. Geol. Sinica, 31, 63-71, 1996

Shu, L. S., R. S. Ma, L. Z. Guo, and J. Q. Sun, Research on the thrust tectonics of the eastern Tianshan belt, Xinjiang, Sci. Geol. Sinica, 32, 337-350, 1997.

Shu, L. S., J. Charvet, and R. S. Ma, Study of a largescale Paleozoic dextral strike-slip ductile shear zone along the northern margin of the central Tianshan, Xinjiang, Xinjiang Geol., 16, 326-336, 1998.

Shu, L. S., J. Charvet, L. Z. Guo, H. F. Lu, and S. Laurent-Charvet, A large-scale dextral ductile strike-slip zone: The Aqqikkudug-Weiya zone along the northern margin of the central Tianshan belt,
Xinjiang, NW China, Acta Geol. Sinica, 73, 148 $162,1999 \mathrm{a}$.

Shu, L. S., Y. S. Shi, H. F. Lu, J. Charvet, and S. Laurent-Charvet, Paleozoic terrane tectonics in northern Tianshan, northwestern China, in Terrane Paths 99 Circum-Pacific Terrane Conference, edited by C. A. Evenchick, G. J. Woodsworth and R. Jongens, pp. 63-65, Geol. Assoc. Can., St. Johns, Newfoundland, Canada, 1999b.

Shu, L. S., Y. T. Chen, H. F. Lu, J. Charvet, S. LaurentCharvet, and D. H. Yin, Paleozoic accretionary terranes in northern Tianshan, NW China, Chin. J. Geochem., 19(3), 193-202, 2000.

Tapponnier, P., and P. Molnar, Active faulting and Cenozoic tectonics of the Tien Shan, Mongolia and Baykal regions, J. Geophys. Res., 84, 3425-3459, 1979

Windley, B. F., M. B. Allen, C. Zhang, Z. Y. Zhao, and G. R. Wang, Paleozoic accretion and Cenozoic redeformation of the Chinese Tien Shan Range, central Asia, Geology, 18, 128-131, 1990.

Xiao, X. C., Y. Q. Tang, J. Y. Li, M. Zhao, Y. M. Feng, and B. Q. Zhu, On the tectonic evolution of the northern Xinjiang, northwest China, Geosci. Xinjiang, 1, 47-69, 1990 .

Xinjiang Bureau of Geology and Mineral Resources, Regional Geology of Xinjiang Uygur Autonomous Region, 841 pp., Geol. Publ., Beijing, 1992.

Zhou, C., and W. T. Dean, Phanerozoic Geology of Northwest China, 251 pp., Science, Beijing, 1996.

J. Charvet and S. Laurent-Charvet, Institut des Sciences de la Terre d'Orléans, Université d'Orléans, BP 6759, 45067 Orléans, France. (sebastien.laurentcharvet@univ-orleans.fr)

P. Monié, UMR 5573, USTL, 34095 Montpellier, France.

L. Shu, Department of Earth Sciences, University of Nanjing, 210093 Nanjing, P. R. China. 